\title{
The internal consistency of the North Sea carbonate system
}

\author{
Lesley A. Salt ${ }^{\mathrm{a}, *}$, Helmuth Thomas ${ }^{\mathrm{b}}$, Yann Bozec ${ }^{\mathrm{c}}$, Alberto V. Borges ${ }^{\mathrm{d}}$, Hein J.W. de Baar ${ }^{\text {a }}$ \\ a Royal Netherlands Institute for Sea Research, Landsdiep 4, 1797 SZ, Texel, Netherlands \\ b Department of Oceanography, Dalhousie University, Halifax B3H 4J1, NS, Canada \\ c Sorbonne Universités, UPMC Univ Paris 06, CNRS, Adaptation et Diversité en Milieu Marin (AD2M UMR7144), Station Biologique de Roscoff, 29680 Roscoff, France \\ ${ }^{\mathrm{d}}$ Unité d'Océanographie Chimique, Institut et Physique B5, Université de Liège, 4000 Liège, Belgium
}

\section{A R T I C L E I N F O}

\section{Article history:}

Received 9 February 2015

Received in revised form 25 November 2015

Accepted 29 November 2015

Available online 15 December 2015

\section{Keywords:}

Carbon dioxide

North Sea

Internal consistency

Total alkalinity

\begin{abstract}
A B S T R A C T
In 2002 (February) and 2005 (August), the full suite of carbonate system parameters (total alkalinity $\left(\mathrm{A}_{\mathrm{T}}\right)$, dissolved inorganic carbon (DIC), $\mathrm{pH}$, and partial pressure of $\mathrm{CO}_{2}\left(\mathrm{pCO}_{2}\right)$ were measured on two re-occupations of the entire North Sea basin, with three parameters $\left(\mathrm{A}_{\mathrm{T}}\right.$, DIC, $\left.\mathrm{pCO}_{2}\right)$ measured on four additional reoccupations, covering all four seasons, allowing an assessment of the internal consistency of the carbonate system. For most of the year, there is a similar level of internal consistency, with $\mathrm{A}_{\mathrm{T}}$ being calculated to within $\pm 6 \mu \mathrm{mol} \mathrm{kg}{ }^{-1}$ using DIC and pH, DIC to $\pm 6 \mu \mathrm{mol} \mathrm{kg}{ }^{-1}$ using $\mathrm{A}_{\mathrm{T}}$ and $\mathrm{pH}, \mathrm{pH}$ to \pm 0.008 using $\mathrm{A}_{\mathrm{T}}$ and $\mathrm{pCO}_{2}$, and $\mathrm{pCO}_{2}$ to $\pm 8 \mu$ atm using DIC and pH, with the dissociation constants of Millero et al. (2006). In spring, however, we observe a significant decline in the ability to accurately calculate the carbonate system. Lower consistency is observed with an increasing fraction of Baltic Sea water, caused by the high contribution of organic alkalinity in this water mass, not accounted for in the carbonate system calculations. Attempts to improve the internal consistency by accounting for the unconventional salinity-borate relationships in freshwater and the Baltic Sea, and through application of the new North Atlantic salinity-boron relationship (Lee et al., 2010), resulted in no significant difference in the internal consistency.
\end{abstract}

(c) 2016 Elsevier B.V. All rights reserved.

\section{Introduction}

Increasing atmospheric $\mathrm{CO}_{2}$ has been partly mitigated by oceanic uptake, which currently accounts for $25-30 \%$ of the total $\mathrm{CO}_{2}$ emissions (Canadell et al., 2007; Khatiwala et al., 2009; Le Quéré et al., 2015; Sabine et al., 2004). The uptake of $\mathrm{CO}_{2}$ in surface waters causes a shift in the chemistry of seawater, notably increases in $\left[\mathrm{H}^{+}\right]$and a decrease in $\left[\mathrm{CO}_{3}^{2-}\right]$ leading to the phenomenon known as ocean acidification (OA) (Caldeira and Wickett, 2003, 2005; Raven, 2005). Recent findings of Wootton et al. (2008), Provost et al. (2010), and Borges and Gypens (2010) indicate that ocean acidification is proceeding in coastal areas more rapidly than has been predicted. The associated changes related to $\mathrm{OA}$ have been shown to have a variety of effects on marine phytoplankton (Fabry et al., 2008). The coastal zone is responsible for a disproportionately large amount of primary production per surface area as compared to the open ocean (Wollast, 1998) and is responsible for a similarly large proportion of carbon export (Dunne et al., 2007). As such, understanding how these waters respond to acidification is vital

\footnotetext{
* Corresponding author at: Sorbonne Universités, UPMC Univ Paris 06, CNRS, Adaptation et Diversité en Milieu Marin (AD2M UMR7144), Station Biologique de Roscoff, 29680 Roscoff, France. Tel.: +332982928 17.

E-mail addresses: lesley.salt@sb-roscoff.fr (L.A. Salt), helmuth.thomas@dal.ca (H. Thomas), bozec@sb-roscoff.fr (Y. Bozec), alberto.borges@ulg.ac.be (A.V. Borges), Hein.de.Baar@nioz.nl (H.J.W. de Baar).
}

for accurately predicting the future consequences of continued $\mathrm{CO}_{2}$ increase.

The North Sea has been identified as an effective continental shelfpump of $\mathrm{CO}_{2}$ (Thomas et al., 2004) and has thus undergone intensive study with respect to the $\mathrm{CO}_{2}$ system. High temporally resolved datasets are necessary to understand the large, observed seasonal variability of the carbonate system in the North Sea (Bozec et al., 2006; Frankignoulle and Borges, 2001; Prowe et al., 2009). The use of voluntary observing ships (VOS) to collect underway $\mathrm{pCO}_{2}$ data have been used extensively in the North Sea (Omar et al., 2010), complemented by measurements of one other of the measurable carbonate variables (total alkalinity $\left(A_{T}\right)$, dissolved inorganic carbon (DIC), and $\mathrm{pH}$ ). From at least two of the measured variables (a combination pair of $A_{T}$, DIC, $\mathrm{pH}$, or $\mathrm{pCO}_{2}$ ), the carbonate system can then be fully solved and quantified using thermodynamic relationships (Park, 1969), which can be integrated in computer programs, such as CO2SYS (Lewis and Wallace, 1998). These calculated parameters, however, are prone to errors, which have been well documented (Millero et al., 1993; Lee et al., 2000; Koeve and Oschlies, 2012; Hoppe et al., 2012). These errors are due to the accuracy of the two measured variables, and to the reliability of the first and second dissociation constants of carbonic acid $\left(K_{1}, K_{2}\right)$. The assessment of the reliability of dissociations constants can be achieved with internal consistency studies.

The first internal consistency studies took place in the open ocean and focused on the reliability of the various sets of carbonic acid 
dissociation constants, which are used to calculate the distribution of inorganic carbon species. Acid dissociation constants describe the interaction between acids (HA), their dissociated proton $(\mathrm{H}+)$, and the conjugate base $(A-)$, shown in Eq. (1).

$\mathrm{K}=\{\mathrm{H}+\}\{\mathrm{A}-\} /\{\mathrm{HA}\}$,

where \{\} denote activities. The aforementioned determinations of $\mathrm{K}_{1}$ and $\mathrm{K}_{2}$ of carbonic acid were performed analytically on artificial seawater (ASW) (Goyet and Poisson, 1989; Hansson, 1973; Roy et al., 1993) and natural seawater (NSW) (Mehrbach et al., 1973) and the data were later refitted using improved computer-generated models (Dickson and Millero, 1987). These constants are technically apparent dissociation constants (denoted by K'), as they are based on concentrations (indicated by []), not activities, so that

$\mathrm{K}^{\prime}=\left\{\mathrm{H}^{+}\right\}\left[\mathrm{A}^{-}\right] /[\mathrm{HA}]$.

This approach also means that the K' values must be determined across the range of salinities, temperatures, and pressures at which they are to be applied and that the measurements take place in the same medium as the solution to which the $\mathrm{K}^{\prime}$ value is to be applied. Assessments and comparisons of each set of constants have taken place in several locations, including the northwest European shelf (Ribas-Ribas et al., 2014), equatorial Pacific (Millero et al., 1993), and the North Atlantic (Lee et al., 1997), and spanning across several ocean basins (Millero et al., 1993,2002; Wanninkhof et al., 1999). At sea studies have been complemented by further work using laboratory set-ups (Lee et al., 1996; Lueker et al., 2000) and more recently investigations into inconsistencies in culture experiments (Hoppe et al., 2012; Koeve and Oschlies, 2012).The conclusions from these studies is that the first and second dissociation constants of carbonic acid $\left(\mathrm{K}_{1}{ }_{1}\right.$ and $\left.\mathrm{K}_{2}\right)$ calculated by Mehrbach et al. (1973) (refit by Dickson and Millero, 1987) are the most reliable when $\mathrm{pCO}_{2}$ is being used in the calculations or is being calculated (Lee et al., 1996; Wanninkhof et al., 1999). These constants were the only ones determined in NSW and the difference in accuracy has been attributed to the lack of boric acid in the ASW (Mojica-Prieto and Millero, 2002). In solution, the majority of dissolved boron (BT $=$ total boron) is present in two forms: boric acid and borate, following the equilibrium relationship below:

$\mathrm{B}(\mathrm{OH})_{3}+\mathrm{H}_{2} \mathrm{O} \leftarrow \mathrm{K}_{\mathrm{B}} \rightarrow \mathrm{B}(\mathrm{OH})_{4}{ }^{-}+\mathrm{H}^{+}$

where $\mathrm{K}_{\mathrm{B}}=\left[\mathrm{B}(\mathrm{OH})_{4}{ }^{-}\right]\left[\mathrm{H}^{+}\right] /\left[\mathrm{B}(\mathrm{OH})_{3}\right]$

In NSW boric acid interacts with $\mathrm{HCO}_{3}^{-}$and $\mathrm{CO}_{3}^{2-}$ to lower the activities, and concentrations of $\mathrm{HCO}_{3}^{-}$, and increase the activities and concentrations of $\mathrm{CO}_{3}^{2-}$, thus lowering $\mathrm{pK}_{1}$ and increasing $\mathrm{pK}_{2}$. Calculations of $\mathrm{pCO}_{2}$ depend directly on the ratio of $\mathrm{K}_{2} / \mathrm{K}_{1}$, thus errors in this ratio will be evident in a lack of internal consistency of $\mathrm{pCO}_{2}$. In contrast, the lack of use of this ratio in calculations with $\mathrm{pH}, \mathrm{A}_{\mathrm{T}}$, or DIC results in other dissociation constants being more appropriate, notably those of Roy et al., (1993) or Goyet and Poisson (1989) (Clayton et al., 1995; Lee et al., 1997). Despite this, in an attempt to homogenize the way carbon chemists carry out these calculations, it is now the recommended standard procedure to use the constants of Mehrbach et al. (1973), refit by Dickson and Millero (1987), on the Total pH scale (Dickson et al., 2007).

Beyond the scope of dissociation constants, additional errors have been associated with systematical differences in equipment (Körtzinger et al., 2000; Ribas-Ribas et al., 2014), high DIC/ $\mathrm{A}_{\mathrm{T}}$ ratios (>1.10) (Lee et al., 1996), the interference of fatty acids (Gripenberg, 1960), and contribution from organic acids and bases to $A_{T}$ (Hernandez-Ayon et al., 2007; Koeve and Oschlies, 2012; Ulfsbo, et al., 2015). The latter errors are only thought to be a problem where dissolved organic carbon
(DOC) reaches particularly high concentrations (Koeve and Oschlies, 2012), thus this is not an issue for open ocean measurements, although presents a major problem for calculations in freshwaters (Abril et al., 2015). Similarly, the contributions of fatty acids and high DIC/ $A_{T}$ ratios in the open ocean are sufficiently low that internal consistency remains within the same order of magnitude as the measurement precision. In culture experiments, the differences between measured and calculated carbonate parameters have been much larger, and thus coastal areas where high primary production takes place could also be susceptible to large discrepancies in calculations of the carbonate system. Furthermore, in the original work of the recommended constants of Mehrbach et al. (1973), the constants were only determined to salinities above 26 . The salinity range was extended in further work by Millero et al. (2006); however, the results have not yet been applied to further internal consistency studies. Coastal regions can also exhibit non-oceanic-like, chemical relationships with salinity, for example, the atypical borate-salinity relationship in the Baltic Sea (Kremling, 1972), which is not taken into account when performing carbonate system calculations and can lead to further error. Here we present the first internal consistency study performed in coastal waters with seasonal resolution over a range of salinities and biogeochemical regimes.

\section{Methods}

\subsection{Hydrography}

The hydrographic properties in the surface waters of the North Sea demonstrate the presence of 3 different water masses, acknowledged in the literature as North Atlantic water, Baltic water, and German Bight water, with the resulting composite water mass known as Central North Sea Water (Bozec et al., 2006; Kempe and Pegler, 1991; Salt et al., 2013). In the northern North Sea (nNS), North Atlantic water flows over the Shetland Shelf bringing characteristic warm and high saline waters into the North Sea. The inflowing water follows an anti-clockwise trajectory around the North Sea, with additional North Atlantic inflow occurring through the English Channel (Otto et al., 1990). In the southeast the Skagerrak water mass, a mixture of Central North Sea water and Baltic water, is easily identifiable through much lower salinities and temperatures than the central North Sea. The German Bight water comes from the coastal region bordering northwest continental Europe in the southeastern North Sea, where the riverine influence is greatest in the North Sea.

The North Sea can be divided into two biogeochemical regimes, within which behavior is determined by depth (Omar et al., 2010; Prowe et al., 2009; Thomas et al., 2005). The nNS (north of $56^{\circ} \mathrm{N}$ ) is deeper than the south (50-400 $\mathrm{m}$ ) and experiences more ocean-like behavior, e.g. higher salinity and seasonal stratification. The southern North Sea (sNS) is much shallower $(<50 \mathrm{~m})$ and remains well-mixed throughout the year. Both regions receive nutrient and organic carbon inputs from terrestrial sources, facilitating high primary production that leads to overall annual autotrophy in the nNS and near balanced trophic status in the sNS (Bozec et al., 2005; Schiettecatte et al., 2007).

\subsection{Measurements and comparisons}

The data used in this study come from 6 separate re-occupations of the North Sea, which took place in August 2001, November 2001, February 2002, May 2002, August 2005, and August 2008. All four carbonate parameters were measured on two of these cruises; February 2002 and August 2005, with a precision of $\pm 2-3 \mu \mathrm{mol} \mathrm{A}_{\mathrm{T}} \mathrm{kg}^{-1}$ for $\mathrm{A}_{\mathrm{T}}$, $\pm 1-2 \mu \mathrm{mol} \mathrm{DIC} \mathrm{kg}{ }^{-1}$ for DIC, \pm 0.001 for $\mathrm{pH}$, and $\pm 1 \mu$ atm for $\mathrm{pCO}_{2}$, respectively. The remaining cruises measured $\mathrm{A}_{\mathrm{T}}$, DIC, and $\mathrm{pCO}_{2}$ with the same level of precision. 


\subsubsection{Carbonate system measurements}

For all of the aforementioned cruises, $A_{T}$ was measured by potentiometric titration to the second end-point $\left(\mathrm{pK}_{2}\right)$ of carbonic acid, using $0.1 \mathrm{M}$ hydrochloric acid as the titrant. For the cruises taking place in August 2001, November 2001, February 2002, May 2002, and August 2005 , the samples for $A_{T}$ were filtered on GF/F shortly after sampling, prior to analysis. The resultant acid volumes and corresponding e.m.f values are fitted to a Gran titration plot using least squares (Gran, 1952). An uncertainty of $\pm 2-3 \mu \mathrm{mol} \mathrm{A}_{\mathrm{T}} \mathrm{kg}^{-1}$ was found as the combined precision for all cruises. Samples for DIC were not filtered in order to prevent gas exchange with the atmosphere. Any sediments or particles collected in the samples would likely settle to the bottom of the sample bottle before analysis and thus interference in the measurements should be minimal. All of the DIC data collected in the North Sea was analyzed by the coulometric method of Johnson et al. (1993), with an overall uncertainty of $\pm 1-2 \mu \mathrm{mol} D \mathrm{DI} \mathrm{kg}^{-1}$ for all cruises. For quality control and calibration of both DIC and $\mathrm{A}_{\mathrm{T}}$, Certified Reference Material (CRM), of known DIC and $A_{T}$, acquired from Prof. Andrew Dickson (Scripps Institute of Oceanography, USA) were used.

In 2002 and 2005, discrete samples were taken from the CTD and analyzed for $\mathrm{pH}$ potentiometrically on the total hydrogen ion scale, calibrated using TRIS (2-amino-2-hydroxymethyl-1,3-propanediol) and AMP (2-aminopyridine) buffers prepared at a salinity of 35 according to Dickson (1993). The accuracy of this method is strongly dependent on the sensitivity of the $\mathrm{pH}$ meter and how accurately the two buffer solutions are made up. For the measurements used in this work, a precision of $\pm 0.001 \mathrm{pH}$ units was attained. For all North Sea cruises, $\mathrm{pCO}_{2}$ was measured continuously, underway, from surface waters, providing the greatest coverage of data. The instrument used continuous equilibration with a headspace and infrared detection with an accuracy of $\pm 1 \mu$ atm (Körtzinger et al., 1996). The calibration of $\mathrm{pCO}_{2}$ measurements was carried out regularly (every 2-3 hours) using National Oceanic and Atmospheric Administration (NOAA, Global Monitoring Division, Carbon Cycle Greenhouse Gases Group) calibrated gas standards. For all cruises, a minimum of 3 calibration gasses were used, spanning a concentration range from 0 to $750 \mathrm{ppm}$.

The concentrations of silicate and phosphate are also required to account for their contribution to $A_{T}$, which can be of the order of up to $1 \mu \mathrm{mol} \mathrm{A} \mathrm{A}_{\mathrm{T}} \mathrm{kg}^{-1}$ in the North Sea. The inorganic nutrients nitrate $\left(\mathrm{NO}_{3}\right)$, ammonium $\left(\mathrm{NH}_{4}\right)$, dissolved silicate $\left(\mathrm{H}_{4} \mathrm{SiO}_{4}\right)$, and phosphate $\left(\mathrm{PO}_{4}\right)$ were analyzed following the methods of Grasshof et al. (1983). Reference nutrient samples (RMNS Kanso, Japan) were analyzed in every run as a control, giving a precision of $\pm 0.2,0.1,0.2$, and 0.01 for the four nutrients, respectively, for each cruise. The salinity and temperature values were recorded by CTD (conductivity-temperature-depth) and salinity values were calibrated post-cruise to salinity samples taken onboard.

\subsection{Calculations}

The internal consistency was assessed using the measured in-situ salinity, temperature, silicate, phosphate, and pressure values. The $\mathrm{KSO}_{4}$ of Dickson (1990) is used and $\mathrm{pH}$ values are given throughout on the total hydrogen ion scale. Not all station positions were occupied and measured in all four seasonal cruises that are compared here. In order to overcome this, the percentage of data points considered internally consistent is often used and given in Tables 3 and 4, to allow direct comparison between the seasons without the loss of data. The calculations were performed using the CO2SYS program (Lewis and Wallace, 1998), adapted for MATLAB (van Heuven, 2011; http://cdiac.ornl.gov/ftp/ co2sys/CO2SYS_calc_MATLAB_v1.1/). All calculated parameters as a function of two input parameters were explored, thus consisting of $A_{T}$ (DIC, pH), $\mathrm{A}_{\mathrm{T}}\left(\mathrm{DIC}, \mathrm{pCO} \mathrm{CO}_{2}\right), \mathrm{A}_{\mathrm{T}}(\mathrm{pH}, \mathrm{pCO})_{2}$, $\mathrm{DIC}\left(\mathrm{A}_{\mathrm{T}}, \mathrm{pH}\right), \operatorname{DIC}\left(\mathrm{A}_{\mathrm{T}}, \mathrm{pCO}_{2}\right)$, DIC $\left(\mathrm{pH}, \mathrm{pCO}_{2}\right), \mathrm{pH}\left(\mathrm{DIC}, \mathrm{A}_{\mathrm{T}}\right), \mathrm{pH}(\mathrm{DIC}, \mathrm{pCO} 2), \mathrm{pH}\left(\mathrm{A}_{\mathrm{T}}, \mathrm{pCO}_{2}\right), \mathrm{pCO}_{2}$ (DIC, $\left.A_{\mathrm{T}}\right), \mathrm{pCO}_{2}(\mathrm{DIC}, \mathrm{pH})$, and $\mathrm{pCO}_{2}\left(\mathrm{~A}_{\mathrm{T}}, \mathrm{pH}\right)$, for the following seven different sets of carbonic acid dissociation constants: Roy et al.
(1993)(R), Goyet and Poisson (1989)(GP), Hansson (1973), refit by Dickson and Millero (1987)(H), Mehrbach et al. (1973), refit by Dickson and Millero (1987)(M), Hansson (1973) and Mehrbach et al. (1973), refit by Dickson and Millero (1987)(HM), Mojica-Prieto and Millero (2002)(MM), and Millero et al. (2006)(ML). In the remaining text, for simplicity, the constants will be referred to by the abbreviations ( $R, G P, H, M, H M, M M$, and ML). The parameterizations of these constants, along with the medium and salinity ranges for which they were determined, are shown in Table 1.

\subsection{Assessment}

The internal consistency of the carbonate system can be considered in a number of different ways. Here, we assess how accurate and precise it is by comparing measured parameters to calculated parameters. Comparing the measured and calculated values, an accurate but imprecise system would be indicated by an average difference $(\Delta)$ close to 0 , but with a high level of variance, denoted by the standard deviation $(\sigma)$. A precise but inaccurate system, however, is indicated by a small $\sigma$, but with a bias, indicated by an average $\Delta$ varying from 0 . Thus, an inaccurate and imprecise system would have a high standard deviation and a large average $\Delta$, while an accurate and precise system would show the inverse. The measured and calculated values of each parameter, for each set of apparent dissociation constants, was fitted to the form $\mathrm{Y}=\mathrm{a} . \mathrm{X}+\mathrm{b}$, by means of total least squares using Matlab 2012 ${ }$, where $\mathrm{X}$ is the calculated value and $\mathrm{Y}$ is the observed value. By fitting the data using total least squares, we take into account the errors in both the $\mathrm{x}$ and $\mathrm{y}$ variables.

\subsection{Error propagation}

When calculating the carbonate parameters in CO2SYS, the uncertainties associated with the observations and calculations are carried through (propagated). Errors are introduced via the measurement techniques of the input parameters, the measurement techniques of the output parameters against which we compare our calculations, and the determinations of the $K_{1}$ and $K_{2}$ values of carbonic acid. The measurement errors carried through in the calculations depend on the input parameter combinations. These margins vary insignificantly over the range of conditions (e.g. temperature, salinity) observed in the North Sea. The error propagation for each calculated parameter, with all variations of input parameter, was calculated following Eq. (5).

$(\varepsilon z)^{2}=\{\delta \mathbf{z} / \delta \mathbf{x}\}^{2} \cdot(\varepsilon \mathrm{x})^{2}+\{\delta \mathrm{z} / \delta \mathrm{y}\}^{2} \cdot(\varepsilon \mathrm{y})^{2}$

The partial derivatives were determined seasonally, using average temperature and salinity values, and determined over the range of values observed. The determined errors for each set of input parameters for each season are given in brackets in the standard deviation section of Table $2 \mathrm{a}$ and $2 \mathrm{~b}$. The error calculation performed here does not take into account errors in the acid dissociation constants, as we later compare the performance of these constants. When the term "internally consistent" is used, it refers to whether the observed values and associated measurement errors fit within the limits of the calculated propagated error.

\subsection{Total borate calculations}

To independently calculate the total borate contribution from each water mass, the individual water mass fractions were first calculated, following Friis et al. (2003). To calculate the Elbe contribution, we used the salinity and $\mathrm{A}_{\mathrm{T}}$ data from the stations closest to the German Bight (at latitudes $>54.4^{\circ} \mathrm{N}$ and longitudes $>8.0^{\circ} \mathrm{E}$ ) to form a regression line with the Central North Sea end member. We then extrapolated this to a salinity of 0 to get a representative freshwater end member. This assumes that all the fresh water present comes from the same source, 
Table 1

Table of parameterizations of the carbonic acid dissociation constants, with temperature and salinity range for each set, and the medium in which measurements were performed.

\begin{tabular}{|c|c|c|c|c|}
\hline Constants & $\mathrm{pK}_{1}$ and $\mathrm{pK}_{2}$ parameterization: & Temperature range & Salinity range & Media \\
\hline $\mathbf{R}$ & $\begin{array}{l}\operatorname{lnK}_{\mathbf{1}}=2.83655-2307.1266 / \mathbf{T}-1.5529413 . \ln (\mathbf{T})+(-0.20760841-4.0484 / \mathbf{T}) . \mathbf{S}^{0.5}+ \\
0.08468345 . \mathbf{S}-0.00654208 . \mathbf{S}^{1.5} \\
\operatorname{lnK}_{\mathbf{2}}=-9.226508-3351.6106 / \mathbf{T}-0.2005743 . \ln (\mathbf{T})+(-0.106901773-23.9722 / \mathbf{T}) . \mathbf{S}^{0.5}+ \\
0.1130822 . \mathbf{S}-0.00846934 . \mathbf{S}^{1.5}\end{array}$ & $0-45$ & $5-45$ & Artificial SW \\
\hline GP & $\begin{array}{l}\mathbf{p K}_{\mathbf{1}}=812.27 / \mathbf{T}+3.356-0.00171 . S \cdot \ln (\mathbf{T})+0.000091 . \mathbf{S}^{2} \\
\mathbf{p K}_{\mathbf{2}}=1450.87 / \mathbf{T}+4.604-0.00385 . S \cdot \ln (\mathbf{T})+0.000182 . \mathbf{S}^{2}\end{array}$ & -1 to 40 & $10-50$ & Artificial SW \\
\hline $\mathbf{H}$ & $\begin{array}{l}\mathbf{p K}_{\mathbf{1}}=851.4 / \mathbf{T}+3.237-0.0106 . \mathbf{S}+0.000105 . \mathbf{S}^{2} \\
\mathbf{p K}_{\mathbf{2}}=-3885.4 / \mathbf{T}+125.844-18.141 . \ln (\mathbf{T})-0.0192 . \mathbf{S}+0.000132 . \mathbf{S}^{2}\end{array}$ & $2-35$ & $20-40$ & Artificial SW \\
\hline M & $\begin{array}{l}\mathbf{p K}_{\mathbf{1}}=3670.7 / \mathbf{T}-62.008+9.7944 . \ln (\mathbf{T})-0.0118 . \mathbf{S}+0.000116 . \mathbf{S}^{2} \\
\mathbf{p K}_{\mathbf{2}}=1394.7 / \mathbf{T}+4.777-0.0184 . \mathbf{S}+0.000118 . \mathbf{S}^{2}\end{array}$ & $2-35$ & $26-43$ & Natural SW \\
\hline HM & $\begin{array}{l}\mathbf{p K}_{\mathbf{1}}=845 / \mathbf{T}+3.248-0.0098 . \mathbf{S}+0.000087 . \mathbf{S}^{2} \\
\mathbf{p K}_{\mathbf{2}}=1377.3 / \mathbf{T}+4.824-0.0185 . \mathbf{S}+0.000122 . \mathbf{S}^{2}\end{array}$ & $2-35$ & $20-40$ & Artificial SW \\
\hline MM & $\begin{array}{l}\mathbf{p K}_{\mathbf{1}}=-43.6977-0.0129037 . \mathbf{S}+1.364 \mathrm{e}^{-4} \cdot \mathbf{S}^{2}+2885.378 / \mathbf{T}+7.045159 \cdot \ln (\mathbf{T}) \\
\mathbf{p K}_{\mathbf{2}}=-452.0940+13.142162 . \mathbf{S}-8.101 \mathrm{e}^{-4} \cdot \mathbf{S}^{2}+21263.61 / \mathbf{T}+68.483143 . \ln (\mathbf{T})+ \\
\left(-581.4428 . \mathbf{S}+0.259601 . \mathbf{S}^{2} / \mathbf{T}-1.967035 . \mathbf{S} \cdot \ln (\mathbf{T})\right.\end{array}$ & $0-45$ & $5-42$ & Natural SW \\
\hline ML & $\begin{array}{l}\mathbf{p K}_{\mathbf{1}}=-126.34048+6320.813 / \mathbf{T}+19.568224 \cdot \ln (\mathbf{T})+13.4038 . \mathbf{S}^{0.5}+0.03206 . \mathbf{S}-5.242 \mathrm{e}^{-5} \cdot \mathbf{S}^{2}+ \\
\left(-530.659 . \mathbf{S}^{0.5}-5.8210 . \mathbf{S}\right) / \mathbf{T}+\left(-2.0664 . \mathbf{S}^{0.5}\right) \cdot \ln (\mathbf{T}) \\
\mathbf{p K}_{\mathbf{2}}=-90.18333+5143.692 / \mathbf{T}+14.613358 \cdot \ln (\mathbf{T})+21.3728 . \mathbf{S}^{0.5}+0.1218 . \mathbf{S}-3.688 \mathrm{e}^{-4} \cdot \mathbf{S}^{2}+ \\
\left(-788.289 . \mathbf{S}^{0.5}-19.189 . \mathbf{S}\right) / \mathbf{T}+\left(-3.374 . \mathbf{S}^{0.5}\right) \cdot \ln (\mathbf{T})\end{array}$ & $0-50$ & $1-50$ & Natural SW \\
\hline
\end{tabular}

however, due to other rivers in the region having a similar catchment lithology, we assume that the chemical signal of the water will be similar. This resulted in a 0 -salinity end member of $2616 \mu \mathrm{mol} \mathrm{A}_{\mathrm{T}} \mathrm{kg}^{-1}$ in February 2002, and $1678 \mu \mathrm{mol} \mathrm{A}_{\mathrm{T}} \mathrm{kg}^{-1}$ in August 2005. These values comparing well with recent seasonal $A_{T}$ data from the Elbe (Amann et al., 2012), which reports $A_{T}$ values of $\sim 2500 \mu \mathrm{mol} A_{T} k g^{-1}$ in winter and $\sim 1750 \mu \mathrm{mol} \mathrm{A} \mathrm{Ag}^{-1}$ in summer at salinities $<1$, thus we assume our estimation is sufficient for the purpose of the calculation.

The borate alkalinity (BAlk) was then calculated for the water mass end members from total boron concentrations. The total boron concentrations (BT) were calculated from (a) the equations of Barth (1998) Eqs. (6) and(7) for the Elbe (German Bight) salinity-boron relationship, (b) Gripenberg (1960) Eq. (8) for the Baltic salinity-boron ratio, (c) Uppström, (1974) for the North Atlantic ratio and also the revised North Atlantic ratio of Lee et al. (2010). At a salinity of 35, the two seasonal linear relationships of Barth (1998), for summer (September 1994) and winter (February 1995), yield an approximate difference of $6 \mu \mathrm{mol} \mathrm{kg}-1$ in the calculated $\left[\mathrm{B}(\mathrm{OH})_{4}^{-}\right]$. As such, for our calculations, Eq. (6) was used for the calculations of August 2005 (summer), and Eq. (7) for the calculation of February 2002 (winter). The water mass fractions were then multiplied by the theoretical BAlk contribution and summed to obtain the actual BAlk of the sample. All of the following Eqs. (6-9) are given for total borate concentration (BT) in mol kg-1 seawater.

$\mathrm{BT}=(0.000127 / 10.811) \cdot \mathrm{S}+1.66 \mathrm{e}^{-5}($ for September 1994$)$

$\mathrm{BT}=(0.000136 / 10.811) \cdot S+1.57 \mathrm{e}^{-5}($ for February 1995$)$

The Baltic Sea has demonstrated a number of chemical relationships varying from standard ocean behavior. The chlorinity-boron relationship has been documented by Gripenberg, (1960), Dyrssen and Uppstrom (1974), and Kremling (1967), who all noted a positive anomaly in the boron/chlorinity ratio. There is little variation in their findings, with the ratio varying from 0.22 (Kremling, 1967) to 0.244 (Gripenberg, 1960). Here, we use the following equation from the data presented by Gripenberg (1960):

$\mathrm{BT}=(0.000244 / 10.811) \cdot(\mathrm{S} / 1.80655)$

For the North Atlantic fraction, the traditional seawater relationship of Uppström (1974) was used:

$\mathrm{BT}=(0.000232 / 10.811) \cdot(\mathrm{S} / 1.80655)$
Furthermore, Lee et al. (2010) recently found boron-salinity relationships significantly different to those reported by Uppström (1974), which were based on measurements solely from the Pacific Ocean. Here, we also apply this new boron-salinity equation Eq. (10), to test whether this improves our internal consistency in the North Sea.

$\mathrm{BT}=(0.0001336 / 10.811) \cdot S$

\section{Results and discussion}

\subsection{A seasonal comparison}

The sampling program of the North Sea is shown in Fig. 1, with the different distinct, biogeochemical regions labeled. The two seasons in which data were collected of all four measurable carbonate parameters were winter (February) and summer (August). These two data sets allow us to compare the internal consistency in two very different conditions, in terms of values of the carbonate variables, and water temperature. As the North Sea is a temperate coastal sea, it is characterized by high primary production during summer and very low, or no, production in winter. The seasonal cycle is fully assessed in the works of Bozec et al. (2006) and Thomas et al. (2009), thus here the discussion is kept brief. The average DIC in February 2002 was $64 \mu \mathrm{mol} \mathrm{DIC} \mathrm{kg}$ higher than that in August. The average $A_{T}$ changed by just $2 \mu \mathrm{mol}$ $A_{T} k^{-1}$; however, the various contributions of chemical species to $A_{T}$ showed larger variability. The changes in $A_{T}$ contributions in the North Sea from February to August are shown in Figs. 2a and b, respectively. For average conditions in the North Sea, the $\mathrm{HCO}_{3}^{-}$and $\mathrm{CO}_{3}^{2-}$ species were by far the greatest contributors to $\mathrm{A}_{\mathrm{T}}$ supplying $\sim 2212 \mu \mathrm{mol} \mathrm{A} \mathrm{A}_{\mathrm{T}} \mathrm{kg}^{-1}$, followed by borate $\left(\sim 68 \mu \mathrm{mol} \mathrm{A} \mathrm{Ag}^{-1}\right)$, hydroxide $\left(\sim 3 \mu \mathrm{mol} \mathrm{A} \mathrm{kg}^{-1}\right)$, phosphate $\left(\sim 0.07 \mu \mathrm{mol} \mathrm{A} \mathrm{Ag}^{-1}\right)$, and silicate $\left(\sim 0.04 \mu \mathrm{mol} \mathrm{A} \mathrm{A}_{\mathrm{T}} \mathrm{kg}^{-1}\right)$. The largest changes between the summer and winter situation were in carbonate alkalinity $\left(\mathrm{HCO}_{3}^{-}+2^{*} \mathrm{CO}_{3}^{2-}\right)$, which was significantly lower in August, when photosynthesis was ongoing. The contribution of silicate and phosphate were also much smaller then, due to lower concentrations resulting from uptake by phytoplankton. The borate contribution remains the second largest contributor to $\mathrm{A}_{\mathrm{T}}$ for both seasons but was slightly higher in August. Isolating the various contributors to this change, we find that the increases in temperature, salinity, and $\mathrm{pH}$ from February to August all affect an individual increase of $\sim 3 \mu \mathrm{mol} \mathrm{kg}{ }^{-1}$ in $\mathrm{B}(\mathrm{OH})_{4}^{-}$(total increase $\sim 9 \mu \mathrm{mol} \mathrm{kg}{ }^{-1}$ ).

The change in average $\mathrm{pCO}_{2}$, from February 2002 to August 2005, was just $8 \mu \mathrm{atm}$; however, this is not representative of the entire 
Table 2

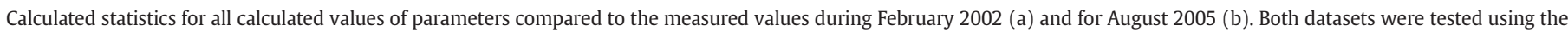

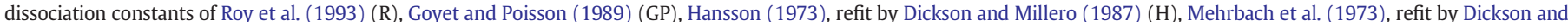
Millero (1987) (M), Hansson (1973) and Mehrbach et al. (1973), refit by Dickson and Millero (1987) (HM), Mojica-Prieto and Millero (2002) (MM), and Millero et al. (2006) (ML).

\begin{tabular}{|c|c|c|c|c|c|c|c|c|c|}
\hline & & & $\mathrm{R}$ & GP & $\mathrm{H}$ & M & $\mathrm{HM}$ & MM & ML \\
\hline \multirow[t]{9}{*}{$A_{T}$} & \multirow[t]{3}{*}{ Regress (slope) } & DIC,pH & 0.999 & 1.000 & 0.998 & 1.001 & 0.999 & 0.999 & 1.001 \\
\hline & & DIC, $\mathrm{pCO}_{2}$ & 0.997 & 0.997 & 0.995 & 1.000 & 0.997 & 0.998 & 0.999 \\
\hline & & $\mathrm{pH}, \mathrm{pCO}_{2}$ & 1.022 & 1.035 & 1.042 & 1.013 & 1.031 & 1.009 & 1.027 \\
\hline & $\mathrm{R}^{2}$ & DIC, $\mathrm{pH}$ & $0.93(<0.01)$ & $0.93(<0.01)$ & $0.93(<0.01)$ & $0.93(<0.01)$ & $0.93(<0.01)$ & $0.93(<0.01)$ & $0.93(<0.01)$ \\
\hline & \multirow[t]{2}{*}{ (p-value) } & DIC, $\mathrm{pCO}_{2}$ & $0.92(<0.01)$ & $0.92(<0.01)$ & $0.93(<0.01)$ & $0.93(<0.01)$ & $0.92(<0.01)$ & $0.92(<0.01)$ & $0.93(<0.01)$ \\
\hline & & $\mathrm{pH}, \mathrm{pCO}_{2}$ & $0.03(0.19)$ & $0.03(0.20)$ & $0.06(0.18)$ & $0.06(0.05)$ & $0.04(0.13)$ & $0.05(0.07)$ & $0.06(0.05)$ \\
\hline & \multirow{3}{*}{$\begin{array}{l}\text { Average } \Delta \text { AT }(\sigma) \\
\left(\mu \mathrm{mol} \mathrm{kg}{ }^{-1}\right)\end{array}$} & DIC, $\mathrm{pH}( \pm 2)$ & $3(8)$ & $1(8)$ & $4(7)$ & $-2(7)$ & $2(8)$ & $3(8)$ & $2(7)$ \\
\hline & & DIC, $\mathrm{pCO}_{2}( \pm 2)$ & $8(9)$ & $8(9)$ & $12(9)$ & $1(8)$ & $8(9)$ & $6(8)$ & $6(8)$ \\
\hline & & $\mathrm{pH}, \mathrm{pCO}_{2}( \pm 43)$ & $-50(63)$ & $-79(62)$ & $-93(62)$ & $-32(60)$ & $-70(61)$ & $-23(61)$ & $48(60)$ \\
\hline \multirow[t]{9}{*}{ DIC } & \multirow[t]{3}{*}{ Regress (slope) } & $\mathrm{TA}, \mathrm{pH}$ & 1.001 & 1.000 & 1.002 & 0.999 & 1.001 & 1.002 & 0.999 \\
\hline & & $\mathrm{TA}, \mathrm{pCO}_{2}$ & 1.003 & 1.003 & 1.005 & 1.000 & 1.003 & 1.002 & 1.001 \\
\hline & & $\mathrm{pH}, \mathrm{pCO}_{2}$ & 1.024 & 1.036 & 1.045 & 1.013 & 1.033 & 1.011 & 1.027 \\
\hline & $\mathrm{R}^{2}$ & $\mathrm{TA}, \mathrm{pH}$ & $0.89(<0.01)$ & $0.90(<0.01)$ & $0.90(<0.01)$ & $0.90(<0.01)$ & $0.90(<0.01)$ & $0.90(<0.01)$ & $0.90(<0.01)$ \\
\hline & \multirow{2}{*}{ (p-value) } & $\mathrm{TA}, \mathrm{pCO}_{2}$ & $0.88(<0.01)$ & $0.88(<0.01)$ & $0.90(<0.01)$ & $0.90(<0.01)$ & $0.89(<0.01)$ & $0.89(<0.01)$ & $0.90(<0.01)$ \\
\hline & & $\mathrm{pH}, \mathrm{pCO}_{2}$ & $0.06(0.07)$ & $0.06(0.07)$ & $0.06(0.05)$ & $0.07(0.03)$ & $0.06(0.06)$ & $0.07(0.04)$ & $0.07(0.04)$ \\
\hline & \multirow{3}{*}{$\begin{array}{l}\text { Average } \Delta \text { DIC }(\sigma) \\
\left.(\mu \mathrm{mol} \mathrm{kg})^{-1}\right)\end{array}$} & $\mathrm{TA}, \mathrm{pH}( \pm 8)$ & $-3(7)$ & $-1(7)$ & $-4(7)$ & $2(7)$ & $-2(7)$ & $-3(7)$ & $-2(7)$ \\
\hline & & $\mathrm{TA}, \mathrm{pCO}_{2}( \pm 7)$ & $-7(8)$ & $-7(8)$ & $-11(7)$ & $-1(7)$ & $-7(8)$ & $-5(7)$ & $6(7)$ \\
\hline & & $\mathrm{pH}, \mathrm{pCO}_{2}( \pm 42)$ & $-50(59)$ & $-76(58)$ & $-92(57)$ & $-29(55)$ & $-68(57)$ & $-25(56)$ & $44(55)$ \\
\hline $\mathrm{pH}$ & Regress (slope) & DIC, $A_{T}$ & 1.001 & 1.000 & 1.001 & 0.999 & 1.000 & 1.001 & 1.000 \\
\hline & & $\mathrm{A}_{\mathrm{T}}, \mathrm{pCO}_{2}$ & 0.999 & 0.998 & 0.998 & 0.999 & 0.998 & 1.000 & 0.999 \\
\hline & & DIC, $\mathrm{pCO}_{2}$ & 0.999 & 0.998 & 0.998 & 0.999 & 0.998 & 0.999 & 0.999 \\
\hline & $\mathrm{R}^{2}$ & DIC, $A_{T}$ & $0.23(<0.01)$ & $0.23(<0.01)$ & $0.25(<0.01)$ & $0.24(<0.01)$ & $0.24(<0.01)$ & $0.25(<0.01)$ & $0.25(<0.01)$ \\
\hline & (p-value) & $\mathrm{A}_{\mathrm{T}}, \mathrm{pCO}_{2}$ & $0.53(<0.01)$ & $0.53(<0.01)$ & $0.53(<0.01)$ & $0.57(<0.01)$ & $0.55(<0.01)$ & $0.56(<0.01)$ & $0.56(<0.01)$ \\
\hline & & DIC, $\mathrm{pCO}_{2}$ & $0.49(<0.01)$ & $0.49(<0.01)$ & $0.49(<0.01)$ & $0.53(<0.01)$ & $0.51(<0.01)$ & $0.52(<0.01)$ & $0.53(<0.01)$ \\
\hline & Average $\Delta \mathrm{pH}(\sigma)$ & DIC, $A_{T}( \pm 0.008)$ & $-0.006(0.017)$ & $0.001(0.017)$ & $-0.008(0.017)$ & $0.004(0.017)$ & $-0.004(0.017)$ & $-0.007(0.017)$ & $0.004(0.017)$ \\
\hline & & $\mathrm{A}_{\mathrm{T}}, \mathrm{pCO}_{2}( \pm 0.001)$ & $0.009(0.011)$ & $0.014(0.011)$ & $0.016(0.011)$ & $0.006(0.010)$ & $0.012(0.011)$ & $-0.004(0.011)$ & $0.011(0.011)$ \\
\hline & & DIC, $\mathrm{pCO}_{2}( \pm 0.001)$ & $0.010(0.012)$ & $0.015(0.012)$ & $0.018(0.012)$ & $0.006(0.011)$ & $0.014(0.011)$ & $0.005(0.011)$ & $0.011(0.011)$ \\
\hline $\mathrm{pCO}_{2}$ & Regress (slope) & DIC, $A_{T}$ & 0.959 & 0.959 & 0.936 & 0.994 & 0.957 & 0.969 & 0.980 \\
\hline & & DIC,pH & 0.975 & 0.963 & 0.956 & 0.985 & 0.967 & 0.987 & 0.973 \\
\hline & & $\mathrm{A}_{\mathrm{T}}, \mathrm{pH}$ & 0.976 & 0.963 & 0.957 & 0.985 & 0.967 & 0.989 & 0.972 \\
\hline & $\mathrm{R}^{2}$ & DIC, $A_{T}$ & $0.14(<0.01)$ & $0.14(<0.01)$ & $0.14(<0.01)$ & $0.17(<0.01)$ & $0.16(<0.01)$ & $0.17(<0.01)$ & $0.18(<0.01)$ \\
\hline & (p-value) & DIC, pH & $0.52(<0.01)$ & $0.52(<0.01)$ & $0.53(<0.01)$ & $0.57(<0.01)$ & $0.54(<0.01)$ & $0.56(<0.01)$ & $0.56(<0.01)$ \\
\hline & & $\mathrm{A}_{\mathrm{T}}, \mathrm{pH}$ & $0.52(<0.01)$ & $0.52(<0.01)$ & $0.53(<0.01)$ & $0.56(<0.01)$ & $0.54(<0.01)$ & $0.55(<0.01)$ & $0.56(<0.01)$ \\
\hline & Average $\Delta \mathrm{pCO}_{2}$ & DIC, $\mathrm{A}_{\mathrm{T}}( \pm 8)$ & $15(18)$ & $15(18)$ & $24(17)$ & $2(17)$ & $16(17)$ & $11(17)$ & $7(17)$ \\
\hline & $(\sigma)(\mu \mathrm{atm})$ & DIC, $\mathrm{pH}( \pm 4)$ & $9(10)$ & $14(11)$ & $17(11)$ & $5(10)$ & $12(10)$ & $5(10)$ & $10(10)$ \\
\hline & & $\mathrm{A}_{\mathrm{T}}, \mathrm{pH}( \pm 3)$ & $9(11)$ & $14(11)$ & $16(11)$ & $6(10)$ & $12(11)$ & $4(10)$ & $10(10)$ \\
\hline $\mathrm{A}_{\mathrm{T}}$ & Regress & DIC, $\mathrm{pH}$ & 0.992 & 0.994 & 0.996 & 0.997 & 0.995 & 0.995 & 0.998 \\
\hline & & DIC, $\mathrm{pCO}_{2}$ & 0.994 & 0.994 & 0.995 & 1.001 & 0.996 & 0.999 & 1.000 \\
\hline & & $\mathrm{pH}, \mathrm{pCO}_{2}$ & 0.977 & 0.991 & 0.999 & 0.962 & 0.988 & 0.960 & 0.976 \\
\hline & $\mathrm{R}^{2}$ & DIC, $\mathrm{pH}$ & $0.96(<0.01)$ & $0.96(<0.01)$ & $0.97(<0.01)$ & $0.97(<0.01)$ & $0.97(<0.01)$ & $0.97(<0.01)$ & $0.97(<0.01)$ \\
\hline & (p-value) & DIC, $\mathrm{pCO}_{2}$ & $0.94(<0.01)$ & $0.94(<0.01)$ & $0.94(<0.01)$ & $0.94(<0.01)$ & $0.94(<0.01)$ & $0.94(<0.01)$ & $0.94(<0.01)$ \\
\hline & & $\mathrm{pH}, \mathrm{pCO}_{2}$ & $0.18(<0.01)$ & $0.19(<0.01)$ & $0.19(<0.01)$ & $0.22(<0.01)$ & $0.22(<0.01)$ & $0.20(<0.01)$ & $0.21(<0.01)$ \\
\hline & Average $\Delta$ AT $(\sigma)$ & DIC, $\mathrm{pH}( \pm 2)$ & $18(7)$ & $14(7)$ & $10(6)$ & $7(6)$ & $11(6)$ & $12(6)$ & $5(6)$ \\
\hline & $\left(\mu \mathrm{mol} \mathrm{kg}{ }^{-1}\right)$ & DIC, $\mathrm{pCO}_{2}( \pm 3)$ & $14(9)$ & $13(9)$ & $11(9)$ & $1(9)$ & $9(9)$ & $3(9)$ & $0(9)$ \\
\hline & & $\mathrm{pH}, \mathrm{pCO}_{2}( \pm 55)$ & $53(52)$ & $19(51)$ & $1(51)$ & $86(52)$ & $28(51)$ & $96(52)$ & $55(51)$ \\
\hline DIC & Regress & $\mathrm{A}_{\mathrm{T}}, \mathrm{pH}$ & 1.008 & 1.006 & 1.004 & 1.003 & 1.005 & 1.005 & 1.002 \\
\hline & & $\mathrm{A}_{\mathrm{T}}, \mathrm{pCO}_{2}$ & 1.006 & 1.005 & 1.004 & 0.999 & 1.004 & 1.001 & 1.000 \\
\hline & & $\mathrm{pH}, \mathrm{pCO}_{2}$ & 0.984 & 0.997 & 1.004 & 0.964 & 0.992 & 0.963 & 0.977 \\
\hline & $\mathrm{R}^{2}$ & $\mathrm{~A}_{\mathrm{T}}, \mathrm{pH}$ & $0.97(<0.01)$ & $0.97(<0.01)$ & $0.97(<0.01)$ & $0.97(<0.01)$ & $0.97(<0.01)$ & $0.97(<0.01)$ & $0.97(<0.01)$ \\
\hline & (p-value) & $\mathrm{A}_{\mathrm{T}}, \mathrm{pCO}_{2}$ & $0.95(<0.01)$ & $0.95(<0.01)$ & $0.96(<0.01)$ & $0.96(<0.01)$ & $0.95(<0.01)$ & $0.96(<0.01)$ & $0.96(<0.01)$ \\
\hline & & $\mathrm{pH}, \mathrm{pCO}_{2}$ & $0.17(<0.01)$ & $0.18(<0.01)$ & $0.18(<0.01)$ & $0.21(<0.01)$ & $0.21(<0.01)$ & $0.19(<0.01)$ & $0.19(<0.01)$ \\
\hline & Average $\Delta \mathrm{AT}(\sigma)$ & $\mathrm{A}_{\mathrm{T}}, \mathrm{pH}( \pm 3)$ & $-16(6)$ & $-13(6)$ & $-9(6)$ & $-6(6)$ & $-10(6)$ & $-11(6)$ & $-4(6)$ \\
\hline & $\left(\mu \mathrm{mol} \mathrm{kg}{ }^{-1}\right)$ & $\mathrm{A}_{\mathrm{T}}, \mathrm{pCO}_{2}( \pm 3)$ & $-12(7)$ & $-11(7)$ & $-9(7)$ & $1(7)$ & $-8(7)$ & $-3(7)$ & $0(7)$ \\
\hline & & $\mathrm{pH}, \mathrm{pCO}_{2}( \pm 36)$ & $33(48)$ & $5(47)$ & $-8(47)$ & $76(48)$ & $16(47)$ & $78(48)$ & $47(47)$ \\
\hline $\mathrm{pH}$ & Regress & DIC, $A_{T}$ & 1.004 & 1.003 & 1.002 & 1.002 & 1.002 & 1.003 & 1.001 \\
\hline & & $\mathrm{A}_{\mathrm{T}}, \mathrm{pCO}_{2}$ & 1.001 & 1.000 & 1.000 & 1.002 & 1.001 & 1.002 & 1.001 \\
\hline & & DIC, $\mathrm{pCO}_{2}$ & 1.001 & 1.000 & 1.000 & 1.002 & 1.000 & 1.002 & 1.001 \\
\hline & $\mathrm{R}^{2}$ & $\mathrm{DIC}, \mathrm{A}_{\mathrm{T}}$ & $0.92(<0.01)$ & $0.92(<0.01)$ & $0.92(<0.01)$ & $0.92(<0.01)$ & $0.92(<0.01)$ & $0.92(<0.01)$ & $0.92(<0.01)$ \\
\hline & (p-value) & $\mathrm{A}_{\mathrm{T}}, \mathrm{pCO}_{2}$ & $0.96(<0.01)$ & $0.96(<0.01)$ & $0.96(<0.01)$ & $0.96(<0.01)$ & $0.96(<0.01)$ & $0.96(<0.01)$ & $0.96(<0.01)$ \\
\hline & & DIC, $\mathrm{pCO}_{2}$ & $0.95(<0.01)$ & $0.95(<0.01)$ & $0.95(<0.01)$ & $0.95(<0.01)$ & $0.95(<0.01)$ & $0.95(<0.01)$ & $0.95(<0.01)$ \\
\hline & Average $\Delta \mathrm{pH}(\sigma)$ & DIC, $A_{T}( \pm 0.007)$ & $-0.031(0.012)$ & $-0.024(0.012)$ & $-0.018(0.012)$ & $-0.012(0.012)$ & $-0.020(0.012)$ & $-0.021(0.012)$ & $-0.009(0.012)$ \\
\hline & & $\mathrm{A}_{\mathrm{T}}, \mathrm{pCO}_{2}( \pm 0.001)$ & $0.009(0.009)$ & $-0.003(0.008)$ & $0.000(0.008)$ & $-0.014(0.008)$ & $-0.005(0.008)$ & $-0.015(0.008)$ & $-0.009(0.008)$ \\
\hline & & DIC, $\mathrm{pCO}_{2}( \pm 0.001)$ & $-0.006(0.009)$ & $-0.001(0.009)$ & $0.002(0.009)$ & $-0.014(0.009)$ & $-0.003(0.009)$ & $-0.015(0.009)$ & $-0.009(0.009)$ \\
\hline $\mathrm{pCO}_{2}$ & Regress & DIC, $A_{T}$ & 0.939 & 0.942 & 0.952 & 1.004 & 0.959 & 0.984 & 1.000 \\
\hline & & DIC, pH & 1.014 & 1.000 & 0.994 & 1.035 & 1.006 & 1.036 & 1.021 \\
\hline & & $\mathrm{A}_{\mathrm{T}}, \mathrm{pH}$ & 1.022 & 1.006 & 0.998 & 1.038 & 1.011 & 1.041 & 1.023 \\
\hline & $\mathrm{R}^{2}$ & DIC, $A_{T}$ & $0.89(<0.01)$ & $0.89(<0.01)$ & $0.89(<0.01)$ & $0.89(<0.01)$ & $0.89(<0.01)$ & $0.89(<0.01)$ & $0.89(<0.01)$ \\
\hline & (p-value) & DIC, pH & $0.96(<0.01)$ & $0.96(<0.01)$ & $0.96(<0.01)$ & $0.96(<0.01)$ & $0.96(<0.01)$ & $0.96(<0.01)$ & $0.96(<0.01)$ \\
\hline & & $\mathrm{A}_{\mathrm{T}}, \mathrm{pH}$ & $0.97(<0.01)$ & $0.97(<0.01)$ & $0.97(<0.01)$ & $0.97(<0.01)$ & $0.97(<0.01)$ & $0.97(<0.01)$ & $0.97(<0.01)$ \\
\hline & Average $\Delta \mathrm{pCO}_{2}(\sigma)$ & DIC, $A_{T}( \pm 6)$ & $22(15)$ & $22(14)$ & $17(14)$ & $-2(13)$ & $15(14)$ & $5(13)$ & $0(13)$ \\
\hline & ( $\mu \mathrm{atm})$ & DIC, $\mathrm{pH}( \pm 4)$ & $-5(8)$ & $-1(8)$ & $2(8)$ & $-12(8)$ & $-2(8)$ & $-13(8)$ & $-8(8)$ \\
\hline & & $\mathrm{A}_{\mathrm{T}}, \mathrm{pH}( \pm 3)$ & $-8(8)$ & $-3(8)$ & $0(8)$ & $-13(8)$ & $-4(8)$ & $-14(8)$ & $-8(8)$ \\
\hline
\end{tabular}


North Sea, as the $\mathrm{pCO}_{2}$ range was two times larger in August (287$455 \mu \mathrm{atm}$ ) compared to February (315-396 $\mu \mathrm{atm}$ ). The range reflected the two biogeochemical regimes within the North Sea: the stratified nNS, where stratification enables prolonged phytoplankton growth causing a large drawdown of $\mathrm{CO}_{2}$, shows very low $\mathrm{pCO}_{2}$ values in August, whereas in the sNS, very low $\mathrm{pCO}_{2}$ values are observed only in spring, and a combination of remineralization and higher temperatures lead to much higher $\mathrm{pCO}_{2}$ in August (Bozec et al., 2005; Schiettecatte et al., 2007; Thomas et al., 2004, 2005). In February, the water column is well-mixed throughout, and biological activity is lower thus leading to a more homogenous surface distribution of $\mathrm{pCO}_{2}$.

The results from all internal consistency calculations using the 4 carbonate parameters, with various dissociation constants, in the February 2002 and August 2005 data sets, are shown in Tables 2a and b, respectively. A regression slope of $>1$ indicates that the measured value is larger than the calculated values. A regression slope of 1.000 is achieved with several combinations in both seasons. Overall, in February, when the three parameters involved are DIC, $\mathrm{A}_{\mathrm{T}}$, and $\mathrm{pCO}_{2}$, (e.g. DIC $\left(\mathrm{A}_{\mathrm{T}}, \mathrm{pCO}_{2}\right), \mathrm{A}_{\mathrm{T}}\left(\mathrm{DIC}, \mathrm{pCO}_{2}\right)$, or $\left.\mathrm{pCO}_{2}\left(\mathrm{DIC}, \mathrm{A}_{\mathrm{T}}\right)\right)$, we find that the constants of $\mathrm{M}$ are the most suitable, whereas when DIC, $\mathrm{A}_{\mathrm{T}}$, and $\mathrm{pH}$ are involved, the constants of GP outperform the others. The largest deviations are consistently observed when the input parameters are $\mathrm{pH}$ and $\mathrm{pCO}_{2}$, with MM producing the most accurate and precise reproduction of measurements. We also observe that the observed standard deviations of differences are largely comparable with the propagation error, with the exception of $\mathrm{pH}$, in which we observe notably larger discrepancies than can be accounted for from the propagation error. In August, the constants of ML are the best choice when the parameter combination is $\mathrm{A}_{\mathrm{T}}$, DIC, and $\mathrm{pCO}_{2}$ and, additionally, when $\mathrm{A}_{\mathrm{T}}$, DIC, and $\mathrm{pH}$ are all involved. However, for calculations using both $\mathrm{pH}$ and $\mathrm{pCO}_{2}$, the constants of GP and $\mathrm{H}$ are the most accurate.

In February and August, the variability in the standard deviations between the dissociation constants (e.g. comparing $\sigma \Delta \mathrm{A}_{\mathrm{T}, \mathrm{R}}$ with $\sigma \Delta \mathrm{A}_{\mathrm{T}, \mathrm{ML}}$ ) were relatively minor compared to the differences obtained with various input parameter combinations (e.g. comparing $\sigma \Delta \mathrm{A}_{\mathrm{T},(\mathrm{pH}, \mathrm{pCO} 2)}$ with $\sigma \Delta \mathrm{A}_{\mathrm{T},(\mathrm{DIC}, \mathrm{pH})}$ etc.). As such, beyond assessing the parameter combinations with the largest degree of noise, the standard deviations are not further discussed in this section. The results for both February and August are consistent, showing that the best regression coefficients for $A_{T}$ were attained with the inputs (DIC, $\left.p C_{2}\right)$, for DIC it was $\left(A_{T}\right.$, $\mathrm{pCO}_{2}$ ), and for $\mathrm{pCO}_{2}$ it was (DIC, $\mathrm{A}_{\mathrm{T}}$ ). For $\mathrm{A}_{\mathrm{T}}$ and DIC, this is in line with smaller error propagations, as similarly found by Dickson and Riley (1974); however, the much improved result for calculating $\mathrm{pCO}_{2}$ using $\left(\mathrm{DIC}, \mathrm{A}_{\mathrm{T}}\right.$ ) is indicative of the improvements in measurement accuracy obtained with these parameters with the use of CRMs. When calculating $\mathrm{pH}$, a regression coefficient of 1.000 was attained with all combination parameters. Similarly, consistency was found in the parameter input combinations resulting in the greatest precision, with

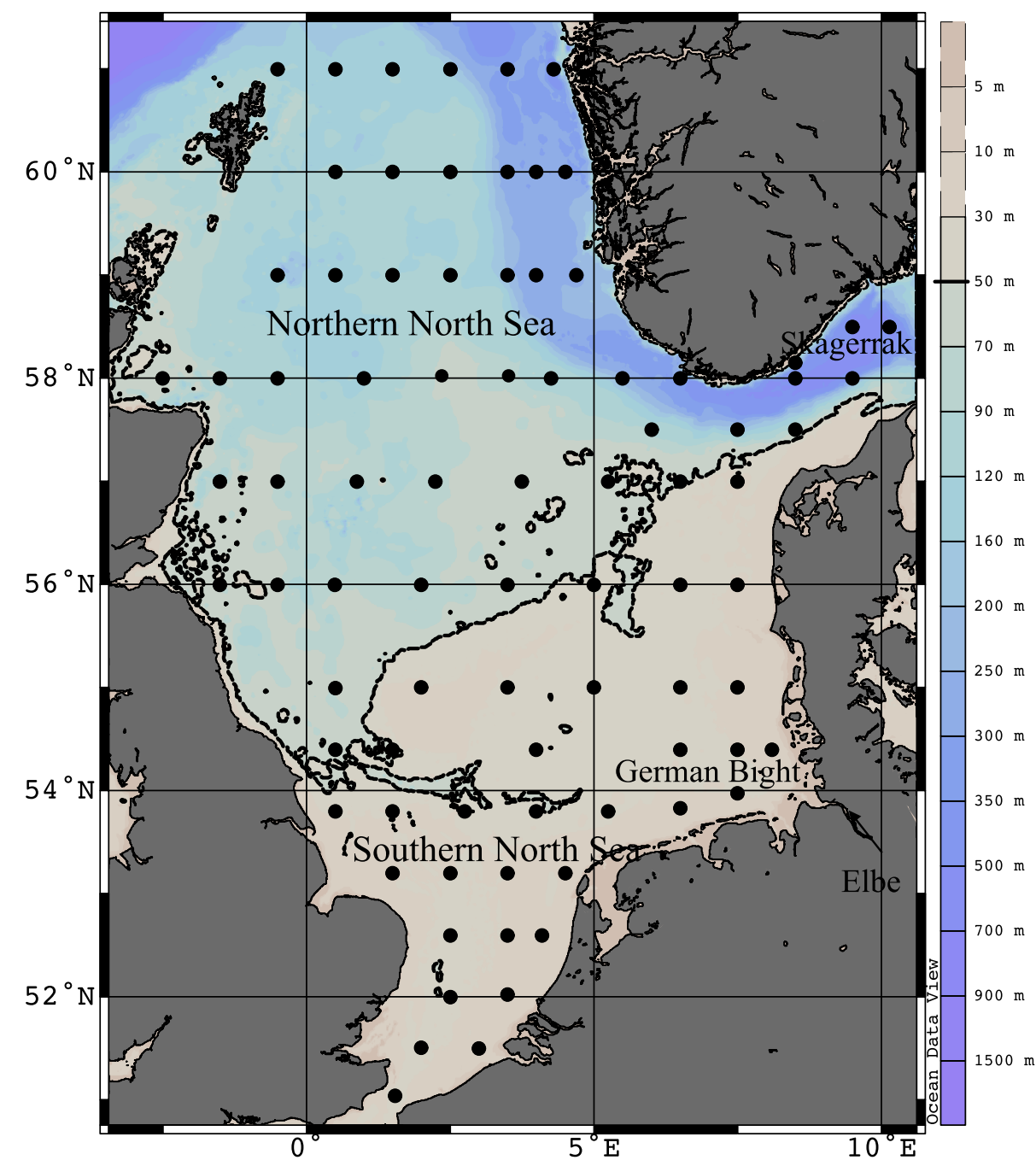

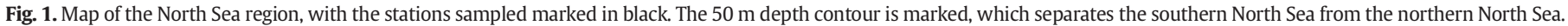


$\mathrm{A}_{\mathrm{T}}$ being best represented by (DIC, pH), DIC by $\left(\mathrm{A}_{\mathrm{T}}, \mathrm{pH}\right), \mathrm{pH}$ by $\left(\mathrm{A}_{\mathrm{T}}\right.$, $\mathrm{pCO}_{2}$ ), and $\mathrm{pCO}_{2}$ by (DIC, $\left.\mathrm{pH}\right)$. The combination pair resulting in the lowest residuals varied between the two seasons, possibly indicating a bias in some of the measurements. The prevalence of better results being obtained when using $\mathrm{pH}$ as an input parameter indicate that these measurements are reliable; however, the difference in the resulting residuals from e.g. $\mathrm{DIC}\left(\mathrm{A}_{\mathrm{T}}, \mathrm{pH}\right)$ and $\mathrm{DIC}\left(\mathrm{A}_{\mathrm{T}}, \mathrm{pCO}_{2}\right)$ are still directly comparable.

The results from February show a slight overall decrease in precision, represented by a higher standard deviation, compared to that obtained in August; however, the average differences show a similar level of accuracy for DIC and $\mathrm{pCO}_{2}$ and a mildly lower bias in February for $\mathrm{A}_{\mathrm{T}}$ and $\mathrm{pH}$. The standard deviations of the differences between the calculated and measured parameters $(\Delta)$ for February 2002 and August 2005 (shown in brackets, respectively) are approximately double to those found in the open ocean by Lee et al. (1997), of $\pm 3 \mu \mathrm{mol} \mathrm{A}_{\mathrm{T}} \mathrm{kg}^{-1}$ in $\mathrm{A}_{\mathrm{T}}\left( \pm 7,6 \mu \mathrm{mol} \mathrm{kg}{ }^{-1}\right), \pm 3 \mu \mathrm{mol}$ DIC kg${ }^{-1}$ in DIC $\left( \pm 7,6 \mu \mathrm{mol} \mathrm{kg}{ }^{-1}\right)$, and $\pm 1.3 \%$ in $\mathrm{pCO}_{2}( \pm 10,8 \mu \mathrm{atm} ; \pm 3 \%, 2 \%)$.

\subsubsection{The effects of salinity on dissociation constants}

Different salinity and temperature parameterizations used in the determination of $\mathrm{pK}_{1}$ and $\mathrm{pK}_{2}$ (Table 1 ) are largely responsible for the deviations in the calculations between dissociation constants. Plotting the difference in $\Delta \mathrm{A}_{\mathrm{T}}$ between results from different dissociation
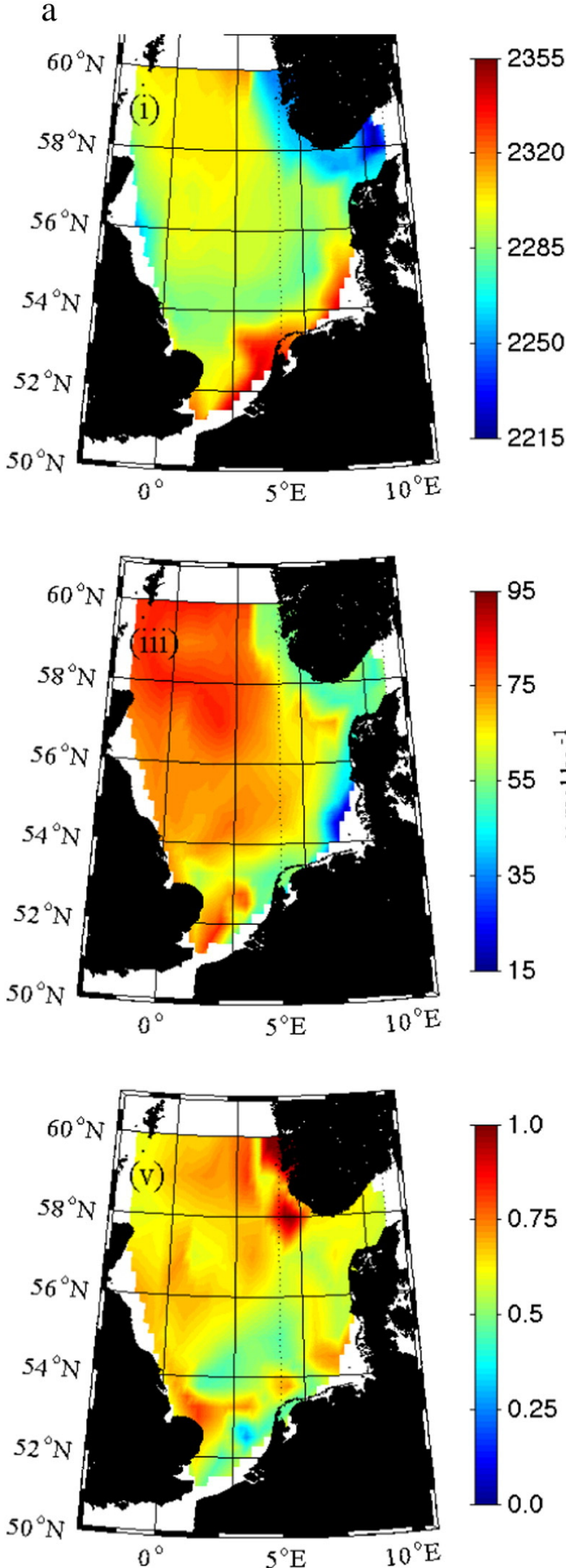
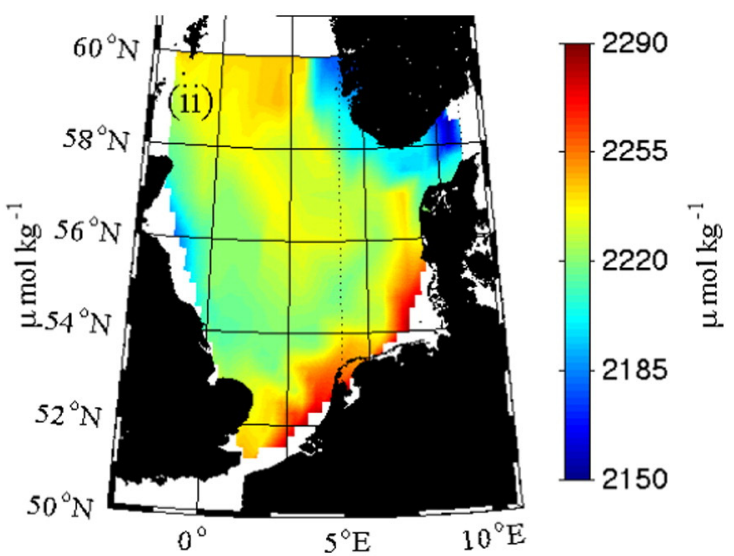

2185

2150
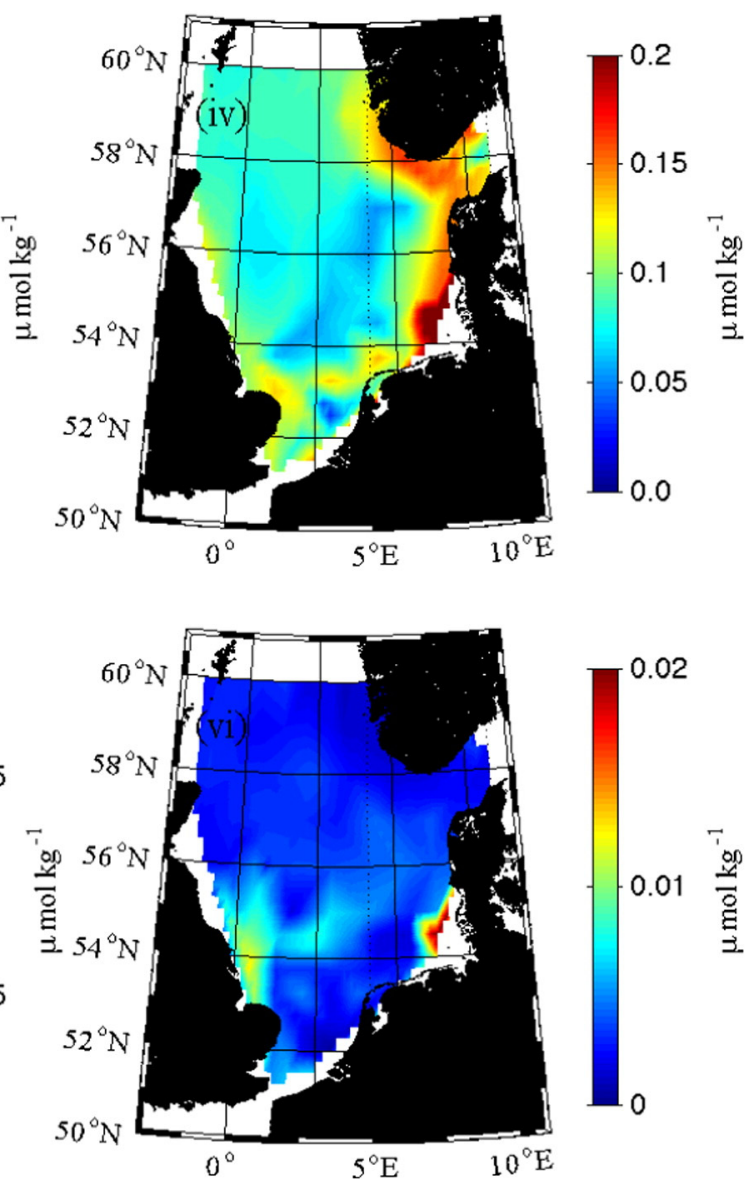

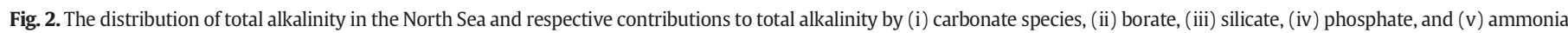
in February 2002 (panel a) and in August 2005 (panel b). 
$\mathrm{b}$
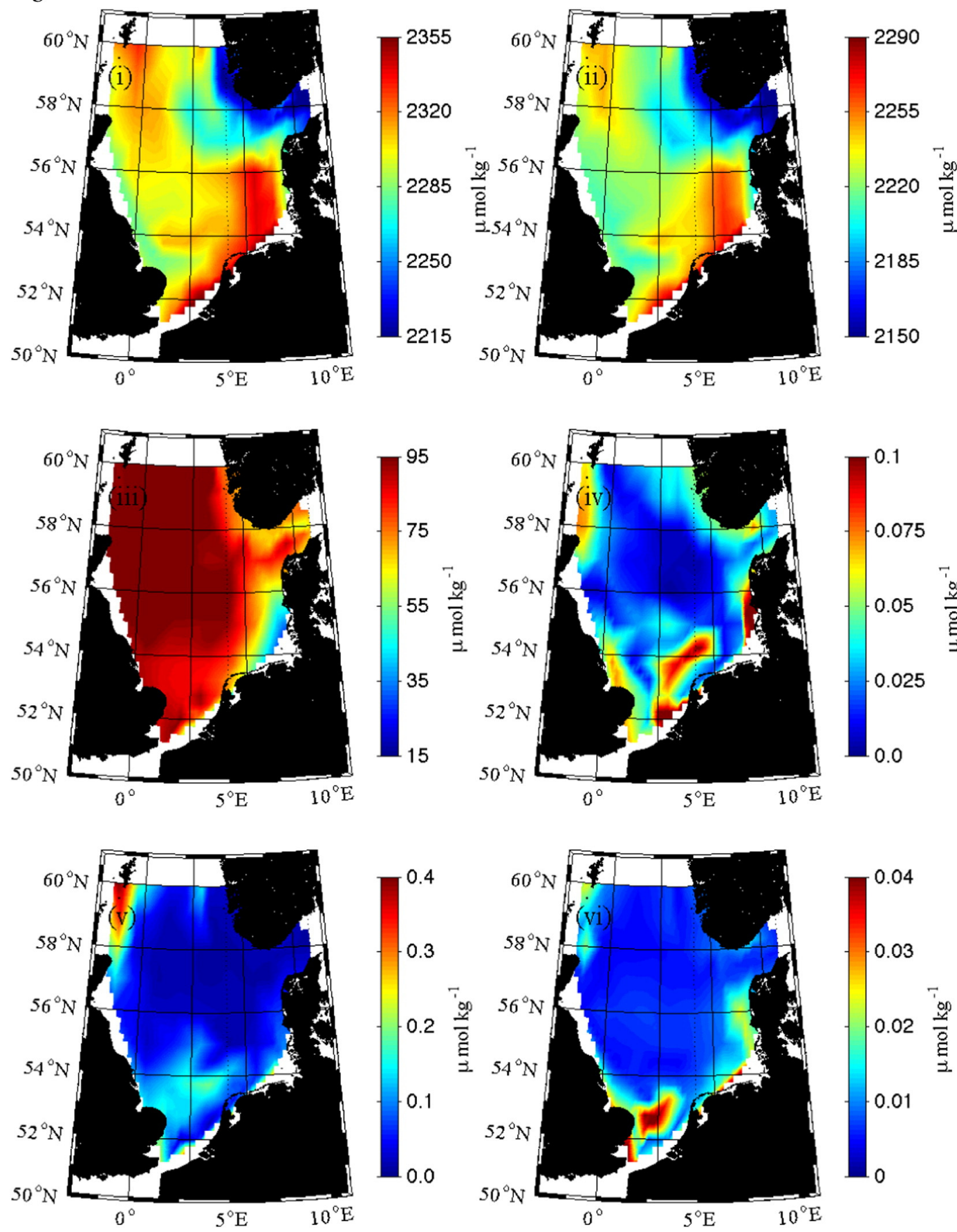

Fig. 2 (continued).

constants (e.g. the differences between the residuals using the constants of $\mathrm{R}$ and the residuals using the constants of $\mathrm{M}: \Delta \mathrm{A}_{T}^{\mathrm{R}}-\Delta \mathrm{A}_{\mathrm{T}}^{\mathrm{M}}$; or $\Delta \mathrm{DIC}$, $\Delta \mathrm{pH}$, and $\Delta \mathrm{pCO}_{2}$ ) shows an exponential-like increase, or decrease, around a salinity of $\sim 34.5$ (Fig. 3), indicating that at higher salinities, the choice of dissociation constants becomes more significant. We compared the differences in residuals of all dissociation constants to those of $\mathrm{M}$ and found the same relationship to that shown in Fig. 3 for the constants of R, GP, $H$, and HM. The differences in $\Delta A_{T}$ between $M$ and $M M$ showed a gradual linear increase with salinity, with a slope of 0.3 $\left(\mathrm{R}^{2}=0.9\right.$, $\mathrm{p}$-value $\left.<0.01\right)$, indicating that across the range of 5 salinity units, there is an increase of $1.5 \mu \mathrm{mol} \mathrm{A} \mathrm{A}_{\mathrm{T}} \mathrm{kg}^{-1}$ difference in $\Delta \mathrm{A}_{\mathrm{T}}$. The residuals resulting from the use of constants ML showed no discernible difference to those of M over the salinity range investigated. At lower salinities, the ratio of $\mathrm{K}_{1} / \mathrm{K}_{2}$ is larger (Mosley et al., 2010; Zeebe and Wolf-Gladrow, 2001) leading to relatively greater $\mathrm{HCO}_{3}^{-}$concentrations compared to $\mathrm{CO}_{2}$ and $\mathrm{CO}_{3}^{2-}$. This ratio is used directly in the calculation of $\mathrm{pH}$ and $\mathrm{A}_{\mathrm{T}}$ from $\mathrm{DIC}$ and $\mathrm{pCO}_{2}$. Due to the different parameterizations of $\mathrm{K}_{1}$ and $\mathrm{K}_{2}$ for the different constants, the difference in this ratio between constants may become larger or smaller at lower salinities. This will lead to larger or smaller differences between the measured and calculated parameters. As such, at lower salinities, one would expect, overall, lower variation between the calculations from the different dissociation constants, as is observed here.

To investigate the performance of the six dissociation constants across a range of different salinities, the North Sea data from August ('05) and February ('02) were split into two groups; low salinity waters, 


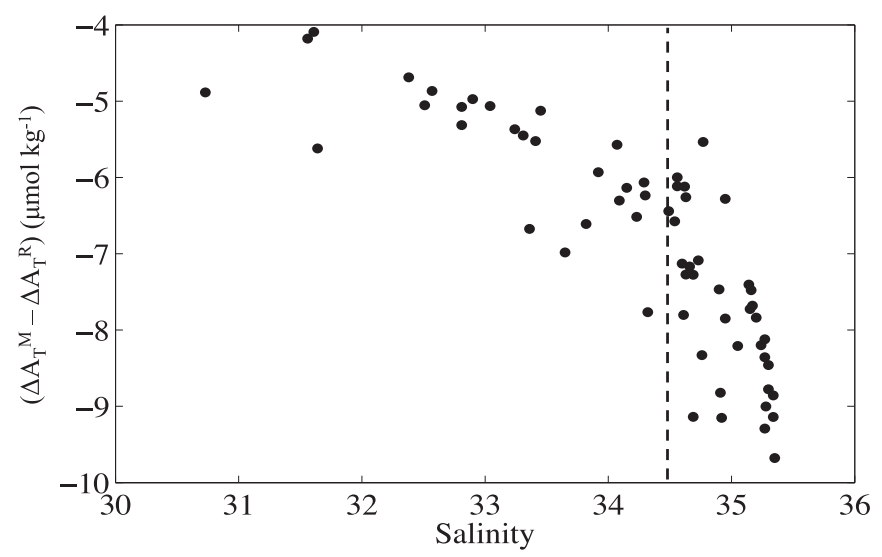

Fig. 3. The difference in $\Delta A_{T}$ ( $\mu \mathrm{mol} A_{T} k^{-1}$ ) across the measured salinity range. The $\Delta A_{T}^{R}$ and $\Delta \mathrm{A}_{\mathrm{T}}^{\mathrm{M}}$ are calculated using (DIC, $\mathrm{pCO}_{2}$ ) as input parameters.

with salinities less than 34.5 , and high salinity waters, with salinities greater than or equal to 34.5. The 1 standard deviation $(\sigma)$ of $\Delta \mathrm{A}_{\mathrm{T}}$, $\triangle \mathrm{DIC}, \triangle \mathrm{pH}$, and $\Delta \mathrm{pCO}_{2}$ is larger, for all parameters and in both seasons, for low salinity waters compared to high salinity waters. In February, in high salinity waters, the standard deviation of $\Delta \mathrm{A}_{\mathrm{T}}$ is $2 \mu \mathrm{mol} \mathrm{A}_{\mathrm{T}} \mathrm{kg}^{-1}$ lower than that of the total dataset; $\triangle \mathrm{DIC}$ is $1 \mu \mathrm{mol} \mathrm{DIC} \mathrm{kg}{ }^{-1}$ lower, $\Delta \mathrm{pH}$ is 0.002 lower and $\Delta \mathrm{pCO}_{2}$ is $2 \mu \mathrm{atm}$ lower. In the less saline waters, there is an increase in the standard deviation of $\Delta \mathrm{A}_{\mathrm{T}}, \Delta \mathrm{DIC}, \Delta \mathrm{pH}$, and $\Delta \mathrm{pCO}_{2}$ by $2 \mu \mathrm{mol} \mathrm{A}_{\mathrm{T}} \mathrm{kg}^{-1}, 1 \mu \mathrm{mol}$ DIC $\mathrm{kg}^{-1}, 0.003$, and $2 \mu \mathrm{atm}$, respectively. The same pattern is observed in August; however the differences are marginally smaller.

Furthermore, in February, there is a clear dichotomy between the different dissociation constants in low and high salinity waters (Fig. 4). As previously mentioned, in winter, when DIC, $\mathrm{A}_{\mathrm{T}}$, and $\mathrm{pH}$ are involved in calculations (that is to say, the calculation of $\mathrm{pH}$ (DIC, $\mathrm{A}_{\mathrm{T}}$ ) or DIC $\left(\mathrm{A}_{\mathrm{T}}, \mathrm{pH}\right)$ or $\mathrm{A}_{\mathrm{T}}(\mathrm{DIC}, \mathrm{pH})$ ), the constants of GP produce the least deviation between measured and calculated parameters. However, we find that in high salinity $(\mathrm{S}>34.5$ ) waters, the constants of $\mathrm{M}$ become more appropriate and in the lower salinity waters $(\mathrm{S}<34.5)$, the constants of R outperform the others. Despite these changes, the constants of MM and ML show little variation in performance between the two salinity environments. For August, there was no difference between the most suitable constants for the entire dataset compared to the different salinity regimes regarding measurements with DIC, $\mathrm{A}_{\mathrm{T}}$, and $\mathrm{pH}$, with the constants of GP still most closely representing the measurements. With regard to measurements of $\mathrm{pH}, \mathrm{pCO}_{2}$, and $\mathrm{DIC}$, however, in the lower salinity waters, the constants of $\mathrm{H}$ outperformed those of GP. Similarly to February, the constants of MM and ML also perform well and show the best consistency across the entire salinity range, with the constants of ML generally displaying a greater level of accuracy. The salinity range does not change between the two seasons; however, in February 2002, a 3.5-fold higher Elbe river discharge was recorded compared to August 2005 (GKSS, 2012, http://coast.gkss.de/staff/kappenberg/elbe/ abfluss/elbe.abfluss). A larger proportion of freshwater in the North Sea could explain why a greater difference is observed between the two salinity environments during this time.

From this application of the various dissociation constants of carbonic acid, we show that the range of salinity directly affects the robustness
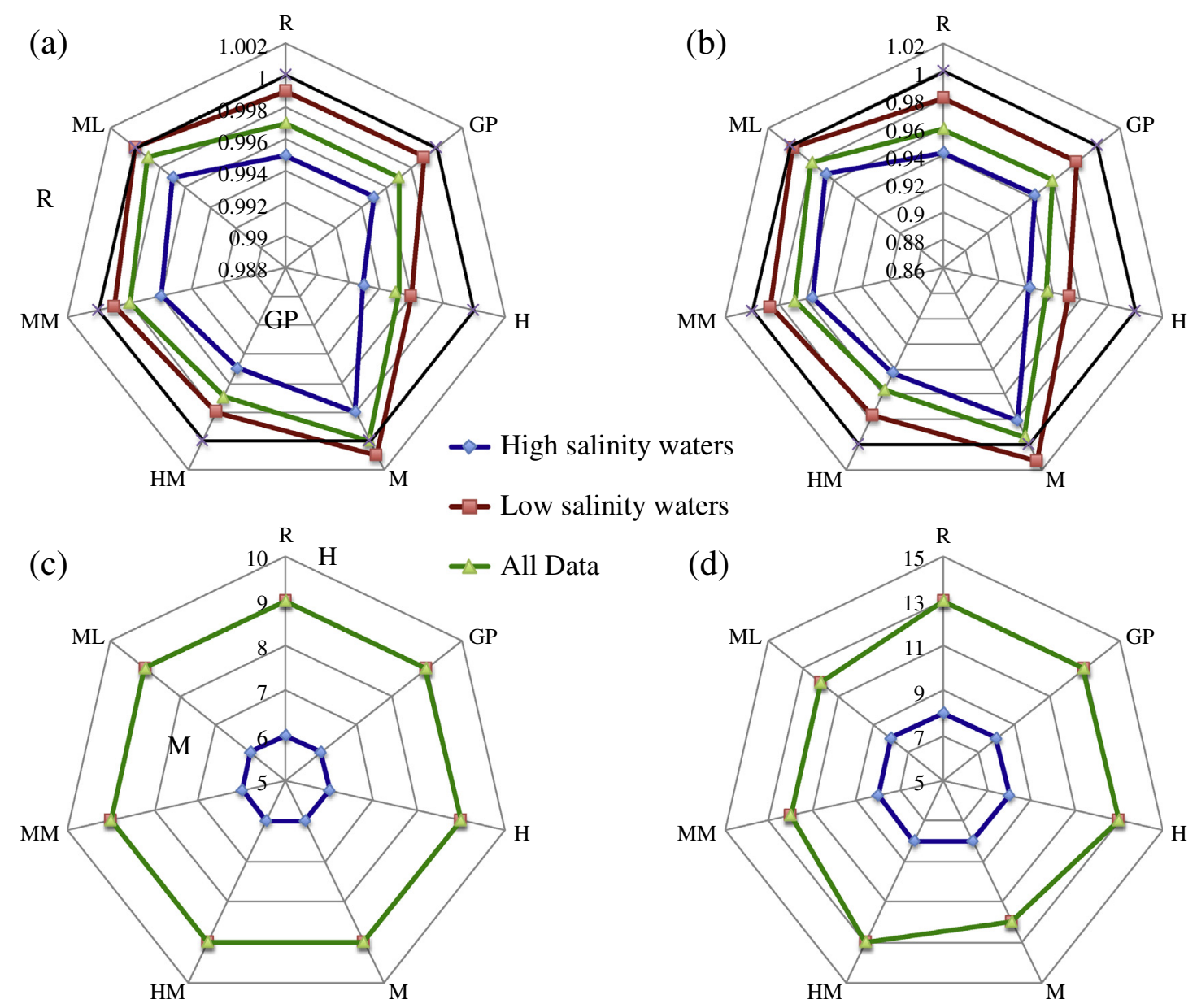

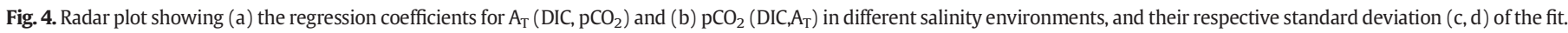


of carbonate system calculations with different constants. Specifically, the constants R, H, GP, and HM show much larger errors in mediums with a larger freshwater constituent compared to those of MM and ML. The larger range of salinities for which these latter constants were determined is likely to contribute to more accurate determinations of $K_{1}$ and $K_{2}$, thus also leading to more accurate $K_{1} / K_{2}$ ratios. Based on these results, for datasets that cover a large range of salinities, for consistency, one can achieve the best overall results using the constants of ML in carbonate system calculations. Despite this, and bearing in mind that the current recommended dissociation constants for carbonate system measurements are those of $\mathrm{M}$, all further calculations and comparisons are performed using this set of constants.

\subsubsection{The role of borate alkalinity}

The ratio of $\mathrm{K}_{1} / \mathrm{K}_{2}$ varies between different parameterizations of carbonic acid dissociation constants, partly depending on whether the constants were determined in artificial seawater (ASW) or natural seawater (NSW). In NSW, interactions between borate and carbonate cause an increase in $K_{1}$ and a decrease in $K_{2}$ (Mojica-Prieto and Millero, 2002), which does not occur in ASW due to the lack of boric acid. As such, we suggest that finding the constants of R, GP, and $\mathrm{H}$ to perform comparably with those of $\mathrm{M}$ in less saline waters may be indicative of the lower borate concentrations in freshwater and thus the errors in $\mathrm{pK}_{2}$, arising from the lack of boric acid in their ASW medium, are minimized. Fig. 2a and b clearly show that borate is the second largest non-carbon contributor to $\mathrm{A}_{\mathrm{T}}$, constituting on average $4 \%$ (Zeebe and WolfGladrow, 2001), thus deviations from the true borate concentration can potentially cause substantial errors in calculations involving $\mathrm{A}_{\mathrm{T}}$. Additionally, the $K_{B}$ of the boric acid system is very close to ocean $\mathrm{pH}$, thus any miscalculation of $\left[\mathrm{H}^{+}\right]$can also lead to significant errors in the calculation of $\left[\mathrm{B}(\mathrm{OH})_{3}\right]$ and $\left[\mathrm{B}(\mathrm{OH})_{4}^{-}\right]$.

Borate has a long residence time in the oceans leading to a conservative relationship with salinity, thus in carbonate system calculations, the total boron contribution is calculated using the linear relationship to chlorinity as found by Uppström (1974), and the borate then calculated using a thermodynamically derived $\mathrm{K}_{\mathrm{B}}$. The data used by Uppström (1974) are based on data obtained from the Pacific Ocean. However, recent findings suggest that this relationship is not consistent in all the world's oceans (Lee et al., 2010). Lee et al. (2010) recently found that this relationship is not as ubiquitous in the oceans as originally thought and has made adjustments which, on provisional tests, led to an increase in the internal consistency of carbonate system calculations in the North Atlantic. The Uppström (1974) borate-salinity relationship was substituted in CO2SYS for the new Lee et al. (2010) relationship, to see if this improved the internal consistency. The new calculations led to a maximum difference in borate alkalinity of $4 \mu \mathrm{mol} \mathrm{A} \mathrm{T}^{-1}$, during both seasons. Despite this change, the influence on internal consistency was almost imperceptible and resulted in no significant improvement.

In the North Sea, the Baltic input through the Skagerrak and the Elbe input in the German Bight have also revealed non-conventional salinity-borate relationships (Barth, 1998; Kremling, 1972). To evaluate the potential error introduced into calculations of the carbonate system in the North Sea by unaccounted-for borate, the borate concentrations in the Elbe- and Baltic-influenced areas of the North Sea were calculated using the water mass fractions and borate-salinity equations from the literature (see Section 2.5). This "pre-calculated" borate contribution to alkalinity (BAlk $\mathrm{pre}_{\mathrm{p}}$ ) was then implemented into CO2SYS, replacing the normal salinity-borate calculation (Uppström, 1974). For comparative purposes, BAlk was also calculated using the traditional Uppström (1974) equation but using various $\left[\mathrm{H}^{+}\right]$determinations, including that from direct $\mathrm{pH}$ measurements, using DIC and $\mathrm{A}_{\mathrm{T}}$ as input parameters and using $\mathrm{A}_{\mathrm{T}}$ and $\mathrm{pCO}_{2}$ as input parameters $\left(\mathrm{DIC} / \mathrm{pCO}_{2}\right.$ produce the same results as $\mathrm{A}_{\mathrm{T}} / \mathrm{pCO}_{2}$, hence have not been included). The BAlk pre calculations showed little change from the BAlk concentrations, the difference showing a normal distribution with 0 as the mean of the absolute values and $\sigma$ of $3 \mu \mathrm{mol} \mathrm{kg}{ }^{-1}$. The equations of Barth (1998) are very similar to the oceanic relationships, thus no significant difference was observed in the German Bight between the two calculations. The greatest difference occurs in the Skagerrak where the Uppström (1974) ratio underestimates the BAlk compared to the equation derived from Gripenberg's data. The differences in calculation resulted in a maximum difference in BAlk of $8 \mu \mathrm{mol} \mathrm{kg}{ }^{-1}$ in August 2005. Despite these

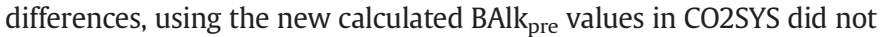
improve the internal consistency; on the contrary, the changes caused greater deviations between the measured and calculated parameters, reducing the number of internally consistent stations for all input combinations. Thus we conclude that the non-conservative salinity-borate relationships in the North Sea are not limiting factors for the internal consistency.

When the $\left[\mathrm{H}^{+}\right]$from pH measurements is used to calculate BAlk directly, and this value is implemented into the thermodynamic calculations, the overall internal consistency is again unimproved. On the other hand, when the BAlk calculated using $\left[\mathrm{H}^{+}\right]$from (DIC, $\mathrm{A}_{\mathrm{T}}$ ) is implemented, the internal consistency improves notably if DIC or $A_{T}$ are being calculated, for both seasons. The number of internally consistent (IC) stations in February and August, for $\mathrm{A}_{\mathrm{T}}(\mathrm{DIC}, \mathrm{pH})$ and $\mathrm{DIC}\left(\mathrm{A}_{\mathrm{T}}, \mathrm{pH}\right)$, increased by $11 \%$ ( 7 stations) and $36 \%$ ( 29 stations). The internal consistency of $\mathrm{A}_{\mathrm{T}}\left(\mathrm{DIC}, \mathrm{pCO} \mathrm{CO}_{2}\right)$ increased by $8 \%$ and $19 \%$ for February and August, respectively, and DIC $\left(\mathrm{A}_{\mathrm{T}}, \mathrm{pCO}_{2}\right)$ increased by $6 \%$ and $16 \%$ stations, respectively. It should be noted that the $\left[\mathrm{H}^{+}\right]$calculated from (DIC, $A_{T}$ ) is only used to calculated the BAlk, whereas the $\left[\mathrm{H}^{+}\right]$used in the other CO2SYS calculations is calculated from the stated input parameters. As such, we can conclude that it is the effect of miscalculation of $\left[\mathrm{H}^{+}\right]$on the BAlk concentration that contributes significantly to a lack of internal consistency for $\left(\mathrm{A}_{\mathrm{T}}, \mathrm{pCO}_{2}\right)$ and $\left(\mathrm{DIC}, \mathrm{pCO}_{2}\right)$. This can be illustrated by Fig. 5(a), where the change in $\triangle A_{T}$ between the measured and calculated values are plotted against the change in $\left[\mathrm{H}^{+}\right]$between that calculated from (DIC, $\mathrm{A}_{\mathrm{T}}$ ) and (DIC, $\left.\mathrm{pCO}_{2}\right)$. When the $\left[\mathrm{H}^{+}\right]$from (DIC, $\mathrm{A}_{T}$ ) is used to calculate BAlk the $\Delta \mathrm{A}_{\mathrm{T}}$ values are shifted toward the $\mathrm{X}$ axis (0), indicative of the improvement in internal consistency. The reason for such a bias is unknown and may be caused by an undetected bias in one of the measured parameters.

\subsection{Seasonal comparison $\left(A_{T}\right.$, DIC, and $\left.p \mathrm{CO}_{2}\right)$}

\subsubsection{Seasonal internal consistency}

The results from the internal consistency study from all 6 data sets, using just three carbonate parameters $\left(\mathrm{A}_{\mathrm{T}}\right.$, DIC, and $\left.\mathrm{pCO}_{2}\right)$, show a clear dominance of the constants of $\mathrm{M}$ and $\mathrm{ML}$ outperforming the others. Both of these sets of constants show comparable results. The results of the seasonal calculations with the use of M constants are given in Table 3. The statistics indicate that the internal consistency of the North Sea follows a seasonal pattern, with May - the most biologically productive time period - producing less consistent results and the rest of the year displaying a good level of consistency. These findings are in agreement with those of Koeve and Oschlies (2012), who similarly noted significant inconsistencies in the carbonate system in waters of high biological activity.

Additionally, the largest fraction of Baltic water found in the Skagerrak is present in May (17\%). Further analysis shows that during this season, there is a significant ( $\mathrm{p}=0.008, \mathrm{R}^{2}=0.55$ ), positive correlation between the Baltic fraction and the deviation between calculated and measured parameters (Fig. 5(b)). Despite this, in May, the internal consistency could also not be improved by changing the BAlk calculation as done above (see Section 3.1.2). However, Gripenberg (1960) concluded, from back titrations of alkalinity, that while borate did not directly affect the accuracy of the $\mathrm{A}_{\mathrm{T}}$ titrations, the interactions between boric acid and organic material could lead to underestimated alkalinity values. Recent work by Ulfsbo et al. (2015) similarly find that in the Baltic, dissolved organic matter can contribute up to $30 \mu \mathrm{mol} \mathrm{A}_{\mathrm{T}} \mathrm{kg}^{-1}$ to alkalinity, thus rendering the $A_{T}$ equation in CO2SYS invalid. It thus seems 

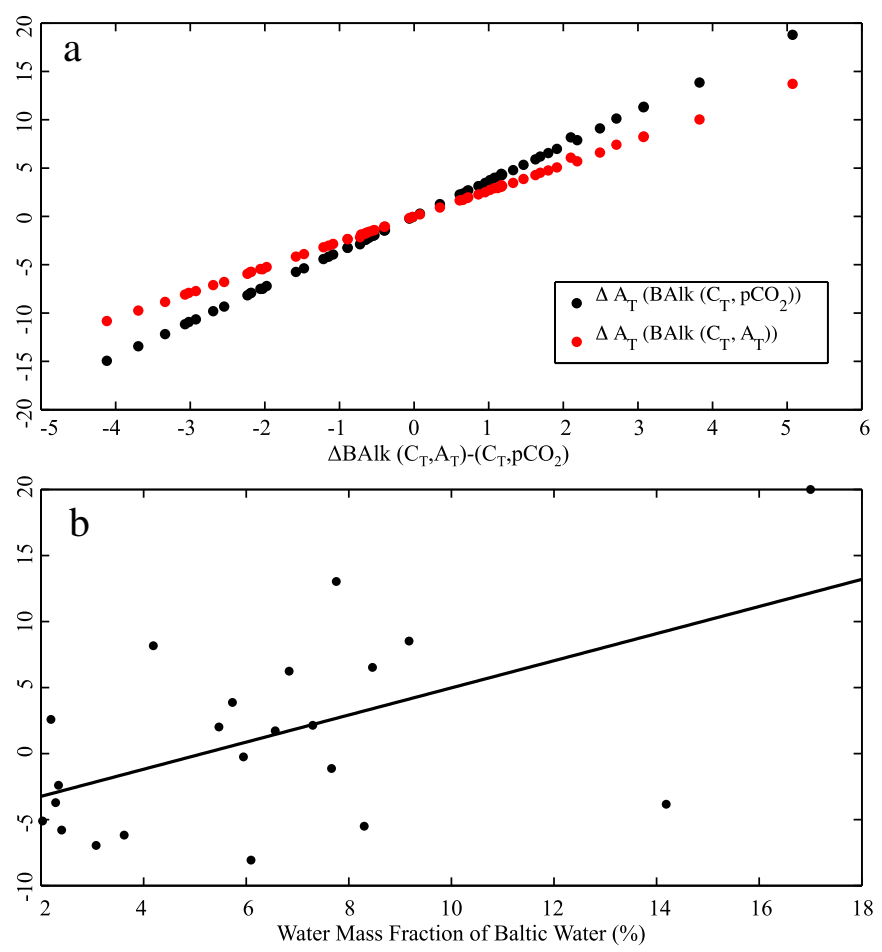

Fig. 5. (a) The change in $\Delta A_{T}$ caused by the difference in BAlk calculated using the $\left[\mathrm{H}^{+}\right]$ from (DIC, $\mathrm{A}_{\mathrm{T}}$ ) (red) and (DIC, $\mathrm{pCO}_{2}$ ) (black). (b) Regression of $\triangle \mathrm{DIC}$ with the calculated water mass fraction of Baltic water, selectively displaying those with a fraction of $2 \%$ or higher.

likely that the lack of internal consistency observed in Baltic-influenced waters in May stems from additional organic material in the water at this time of year, with possibly further errors introduced from the interaction of this organic material with boric acid.

The North Sea receives a high input of allochthonous dissolved organic matter, for which analysis of colored dissolved organic matter (CDOM) indicates that it contains a high proportion of terrigenous sources (Stedmon et al., 2010). Thus may contain terrestrial "titratable" compounds, which are also not accounted for in our calculations. It has been suggested that fatty acids and humic substances may contribute to measured $A_{T}$, and in the Baltic Sea, there is a higher than average concentration of both. The contribution of fatty acids to $A_{T}$ has been fairly extensively examined by Osterroht (1993) and maximum contributions of $<1 \mu \mathrm{mol} \mathrm{A} \mathrm{Ag}_{\mathrm{T}}^{-1}$ to alkalinity were found, which is insufficient to cause the inconsistencies observed here. In contrast, more recently, the presence of humic substances has been shown to contribute to large excess $A_{T}$ in the Baltic Sea (Ulfsbo et al., 2015). The contribution of organic matter to $A_{T}$ has been observed elsewhere, as well. Muller

Table 3

Derived linear regressions, using total least squares, and standard deviations of differences between measured and calculated parameters $\left(\mathrm{A}_{\mathrm{T}}\right.$, DIC, and $\left.\mathrm{pCO}_{2}\right)$ with the percentage of internally consistent stations for all six cruises. The calculations were performed using the acid dissociation constants of Mehrbach et al. (1973), refit by Dickson and Millero (1987).

\begin{tabular}{llllllll}
\hline $\begin{array}{l}\text { Input } \\
\text { combination }\end{array}$ & & $\begin{array}{l}\text { Aug } \\
\text { '01 }\end{array}$ & $\begin{array}{l}\text { Nov } \\
\text { '01 }\end{array}$ & $\begin{array}{l}\text { Feb } \\
\text { '02 }\end{array}$ & $\begin{array}{l}\text { May } \\
\text { '02 }\end{array}$ & $\begin{array}{l}\text { Aug } \\
\text { '05 }\end{array}$ & $\begin{array}{l}\text { Aug } \\
\text { '08 }\end{array}$ \\
\hline $\mathrm{A}_{\mathrm{T}}\left(\mathrm{DIC}, \mathrm{pCO}_{2}\right)$ & Regress & 0.999 & 1.001 & 1.000 & 0.993 & 1.001 & 1.000 \\
& Std & 29 & 8 & 8 & 27 & 9 & 9 \\
& IC $(\%)$ & 15.5 & 47.7 & 43.5 & 9.4 & 34.9 & 50.2 \\
$\mathrm{DIC}\left(\mathrm{A}_{\mathrm{T}}, \mathrm{pCO}_{2}\right)$ & Regress & 1.000 & 0.999 & 1.000 & 1.007 & 1.000 & 1.000 \\
& Std & 24 & 7 & 7 & 23 & 7 & 8 \\
& IC $(\%)$ & 17 & 48 & 44 & 9 & 39 & 52 \\
$\mathrm{pCO}_{2}\left(\mathrm{DIC}, \mathrm{A}_{\mathrm{T}}\right)$ & Regress & 0.976 & 1.004 & 0.994 & 0.906 & 1.004 & 0.998 \\
& Std & 41 & 15 & 17 & 41 & 13 & 15 \\
& IC $(\%)$ & 26 & 50 & 40 & 20 & 49 & 57 \\
\hline
\end{tabular}

and Bleie (2008), using the technique of Hernandez-Ayon et al. (2007) on fjord surface waters, compared the $\mathrm{A}_{\mathrm{T}}$ estimation of DIC to the measured value and found that organic alkalinity can contribute from 2 to $22 \mu \mathrm{mol} \mathrm{A} \mathrm{kg}^{-1}$ in regions of high organic matter. These errors are sufficiently large to account for the noted decrease in internal consistency observed in the North Sea in May, compared to other seasons.

As the North Sea consists of two different biogeochemical regimes, the nNS and the sNS, we investigated whether these two environments contributed toward a difference in the internal consistency of the carbonate system. The North Sea was subsequently divided along the $56^{\circ} \mathrm{N}$ latitude and the internal consistency re-examined in both areas, applying just the constants of $\mathrm{M}$ (Table 4). In May, there is a significant increase in the standard deviation of $\triangle \mathrm{DIC}$ and $\triangle \mathrm{pCO}_{2}$ in the nNS compared to the sNS; however, $\Delta \mathrm{A}_{\mathrm{T}}$ shows no difference. As previously mentioned, there is greater freshwater influence in the sNS. However, the difference observed between the nNS and sNS is larger than the difference previously mentioned between oceanic and estuarine waters. In the nNS, larger blooms of phytoplankton are maintained for long periods due to seasonal, thermal stratification, which is in contrast to the sNS where rapid remineralization takes place in the permanently well-mixed water column. Errors have previously been associated with intense biological activity (Hoppe et al., 2012; Kim and Lee, 2009). Thus, we are inclined to suggest that this may have a similar effect here. However, it is interesting that the errors in $\Delta A_{T}$ are not affected on a north-south latitudinal gradient, like $\triangle \mathrm{pCO}_{2}$ and $\triangle \mathrm{DIC}$.

The different regimes in the nNS and sNS also influence the dominant phytoplankton species present in each location. Calcifying organisms and the presence of coccolithophores are well documented in the nNS, where the development of coccolithophore blooms is controlled by the North Atlantic influence and the stratification of the water column (Burkill et al., 2002; Houghton, 1991). The presence of coccolithophores in waters sampled for DIC could conceivably lead to a degree of $\mathrm{CaCO}_{3}$ dissolution during the DIC titration. This would introduce a degree of noise to the DIC values in the NNS, and possibly account for some of the larger errors observed in this region. Furthermore, Fraga and Alvarez-Salgado (2005) quantified the change in $\mathrm{A}_{\mathrm{T}}$ during photosynthesis and found that the differences are caused predominantly by $\mathrm{CaCO}_{3}$ formation. Calcification influences the $\mathrm{A}_{\mathrm{T}} / \mathrm{DIC}(\mathrm{X})$ ratio of the waters as it removes one mole of DIC per 2 moles of $A_{T}$ (Wolf-Gladrow et al., 2007). Lee et al. $(1996,1997)$ found that high $X$ ratios $(>1.10)$ can impact the sensitivity of $\mathrm{pK}_{2}$, thus affecting the consistency between

Table 4

Calculated internal consistency statistics for all data sets divided into northern North Sea stations (nNS) (latitude $>56^{\circ} \mathrm{N}$ ) and southern North Sea stations (sNS) (latitude $<56^{\circ} \mathrm{N}$ ).

\begin{tabular}{|c|c|c|c|c|c|c|c|c|}
\hline \multirow{3}{*}{$\begin{array}{l}\text { Area } \\
\text { nNS } \\
\quad\left(\text { lat }>56^{\circ} \mathrm{N}\right)\end{array}$} & \multicolumn{2}{|c|}{ Input combination } & \multirow{2}{*}{$\begin{array}{l}\begin{array}{l}\text { Aug } \\
\text { '01 }\end{array} \\
1.00\end{array}$} & \multirow{2}{*}{$\begin{array}{l}\begin{array}{l}\text { Nov } \\
\text { '01 }\end{array} \\
1.00\end{array}$} & \multirow{2}{*}{$\begin{array}{l}\text { Feb } \\
\text { '02 } \\
1.00\end{array}$} & \multirow{2}{*}{$\begin{array}{l}\text { May } \\
\text { '02 } \\
0.99\end{array}$} & \multirow{2}{*}{$\begin{array}{l}\text { Aug } \\
\text { '05 } \\
1.00\end{array}$} & \multirow{2}{*}{$\begin{array}{l}\text { Aug } \\
\text { '08 } \\
1.00\end{array}$} \\
\hline & $\mathrm{A}_{\mathrm{T}}$ & Regress & & & & & & \\
\hline & $\left(\mathrm{DIC}, \mathrm{pCO}_{2}\right)$ & Std & 27 & 8 & 8 & 27 & 9 & 9 \\
\hline & & IC (\%) & 16.0 & 52.8 & 44.7 & 7.4 & 34.8 & 48.1 \\
\hline & DIC & Regress & 1.00 & 1.00 & 1.00 & 1.01 & 1.00 & 1.00 \\
\hline & $\left(\mathrm{A}_{\mathrm{T}}, \mathrm{pCO}_{2}\right)$ & Std & 22 & 7 & 6 & 27 & 7 & 9 \\
\hline & & IC (\%) & 16.0 & 52.8 & 44.7 & 7.4 & 28.3 & 44.4 \\
\hline & $\mathrm{pCO}_{2}$ & Regress & 1.01 & 1.01 & 0.99 & 0.87 & 1.01 & 1.00 \\
\hline & $\left(\mathrm{DIC}, \mathrm{A}_{\mathrm{T}}\right)$ & Std & 30 & 13 & 14 & 48 & 13 & 17 \\
\hline & & IC (\%) & 32 & 55 & 40 & 15 & 46 & 54 \\
\hline & No. stations & & 25 & 53 & 38 & 54 & 46 & 54 \\
\hline \multirow{10}{*}{$\begin{array}{l}\text { sNS } \\
\quad\left(\text { lat } \leq 56^{\circ} \mathrm{N}\right)\end{array}$} & & Regress & 1.00 & 1.00 & 1.00 & 0.99 & 1.00 & 1.00 \\
\hline & $\left(\mathrm{DIC}, \mathrm{pCO}_{2}\right)$ & Std & 29 & 8 & 8 & 27 & 9 & 9 \\
\hline & & IC (\%) & 20.6 & 41.7 & 38.5 & 10.3 & 44.1 & 56.8 \\
\hline & DIC & Regress & 1.00 & 1.00 & 1.00 & 1.00 & 1.00 & 1.00 \\
\hline & $\left(\mathrm{A}_{\mathrm{T}}, \mathrm{pCO}_{2}\right)$ & Std & 24 & 8 & 8 & 12 & 7 & 7 \\
\hline & & IC (\%) & 17.6 & 41.7 & 38.5 & 10.3 & 44.1 & 56.8 \\
\hline & $\mathrm{pCO}_{2}$ & Regress & 0.96 & 1.00 & 1.00 & 0.97 & 1.00 & 1.00 \\
\hline & $\left(\mathrm{DIC}, \mathrm{A}_{\mathrm{T}}\right)$ & Std & 46 & 17 & 19 & 19 & 14 & 15 \\
\hline & & IC (\%) & 23.5 & 47.2 & 38.5 & 25.6 & 52.9 & 59.5 \\
\hline & $\begin{array}{l}\text { No. } \\
\text { stations }\end{array}$ & & 34 & 36 & 26 & 39 & 34 & 37 \\
\hline
\end{tabular}




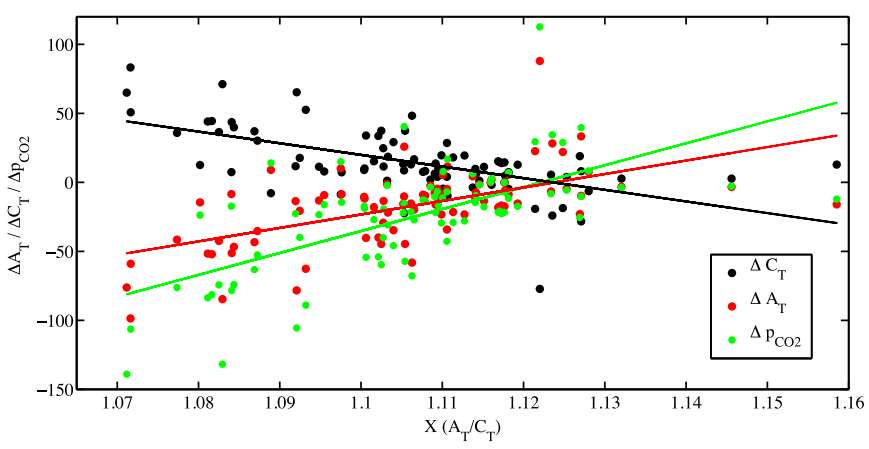

Fig. 6. The change in $\triangle \mathrm{DIC}$ and $\Delta \mathrm{A}_{\mathrm{T}}$ (both in $\mu \mathrm{mol} \mathrm{kg}{ }^{-1}$ ) and $\Delta \mathrm{pCO}_{2}$ (in $\mu$ atm) observed with a varying ratio of $A_{T} / \mathrm{DIC}(\mathrm{X})$ during May 2002.

measured and calculated carbonate parameters. The range of $\mathrm{X}\left(\mathrm{A}_{\mathrm{T}} / \mathrm{DIC}\right)$ found in the North Sea varies from 1.06 to 1.16 throughout the year, with the smallest range (1.06-1.09) occurring in February and the largest range (1.07-1.16) found in May. During May, $X$ also shows a positive linear correlation with $\Delta \mathrm{DIC}, \Delta \mathrm{A}_{\mathrm{T}}$, and $\Delta \mathrm{pCO}_{2}$ with $\mathrm{R}^{2}$ values of $0.6,0.6$, and 0.7 , respectively (Fig. 6 ). The size of the error introduced by varying $\mathrm{X}$ can be up to $5 \mu \mathrm{mol} \mathrm{A}_{\mathrm{T}} \mathrm{kg}^{-1}$, as found by Lee et al. (1996), which would contribute a large proportion of the errors we observe in the North Sea.

\section{Conclusions}

On a basin-wide scale in the North Sea, we find that the carbonic acid dissociation constants of Mehrbach et al. (1973, refit by Dickson and Millero, 1987), and the constants of Millero et al., (2006) provide the best internal consistency. In the case of the former constants, this is in agreement with previous findings (Lee et al., 1996; Wanninkhof et al., 1999). In regions of lower than average ocean salinity, there is markedly less deviation between the internal consistency of the different constants and the constants of ML outperform those of M. At high salinities, these two sets of constants are comparable in performance, thus over datasets with a wide variety of salinities, we recommend the use of ML constants for more consistent results.

In the North Sea in general, we find that the internal consistency of the carbonate system is much less than that found in the open ocean, by approximately 2 -fold, which is unsurprising considering the additional input of terrestrial compounds in coastal areas, and generally higher productivity. Application of the new borate-salinity relationship noted by Lee et al. (2010), and further accounting for the nonconventional salinity-borate relationships in the water masses contributing to the North Sea, did not result in any improvement in the internal consistency. The internal consistency is relatively constant throughout the year, with the exception of May, where there is a marked decrease in our ability to accurately calculate the carbonate system. Waters in which the Baltic Sea constituent is larger than $2 \%$ show lower internal consistency in May driven by the presence of excess organic alkalinity unaccounted for in carbonate system calculations. In the entire North Sea, there is a significant correlation between the internal consistency and $X\left(A_{T} / D I C\right)$, similar to that found in previous studies. Higher $X$ values in the nNS compared to the sNS are likely to contribute to the larger errors observed in this area through the increased sensitivity on $\mathrm{pK}_{2}$.

\section{Acknowledgments}

We thank the captains and crews of the Research Vessel Pelagia and an anonymous reviewer for constructive comments on a previous version of the manuscript. This program was supported by the Research Council for Earth and Life Sciences (ALW) of the Netherlands Organization for Scientific Research (NWO), project number 817.01.004 and contributes to CARBO-OCEAN, an integrated project of the European Union (contract no. 511176-2). This work contributes to IGBP/IHDP LOICZ. AVB is a senior research associate at the FRS-FNRS.

\section{References}

Abril, G., Bouillon, S., Darchambeau, F., Teodoru, C.R., Marwick, T.R., Tamooh, F., Omengo, F.O., Geeraert, N., Deirmendjian, L., Polsenaere, P., Borges, A.V., 2015. Technical note: large overestimation of $\mathrm{pCO}_{2}$ calculated from $\mathrm{pH}$ and alkalinity in acidic, organic-rich freshwaters. Biogeosciences 12 (1), 67-78.

Amann, T., Weiss, A., Hartmann, J., 2012. Carbon dynamics in the freshwater part of the Elbe estuary, Germany: implications of improving water quality. Estuar. Coast. Shelf Sci. 107, 112-121.

Barth, S., 1998. ${ }^{11} \mathrm{~B} /{ }^{10} \mathrm{~B}$ variations of dissolved boron in a freshwater-seawater mixing plume (Elbe Estuary, North Sea). Mar. Chem. 62, 1-14

Borges, A.V., Gypens, N., 2010. Carbonate chemistry in the coastal zone responds more strongly to eutrophication than to ocean acidification. Limnol. Oceanogr. 55, 346-353.

Bozec, Y., Thomas, H., Elkalay, K., de Baar, H.J.W., 2005. The continental shelf pump for $\mathrm{CO}_{2}$ in the North Sea-evidence from summer observation. Mar. Chem. 93, 131-147.

Bozec, Y., Thomas, H., Schiettecatte, L.-S., Borges, A.V., Elkalay, K., de Baar, H.J.W., 2006. Assessment of the processes controlling seasonal variations of dissolved inorganic carbon in the North Sea. Limnol. Oceanogr. 51, 2,746-2,762.

Burkill, P.H., Archer, S.D., Robinson, C., Nightingale, P.D., Groom, S.B., Tarran, G.A., Zubkov, M.V., 2002. Dimethyl sulphide biogeochemistry within a coccolithophore bloom (DISCO): an overview. Deep Sea Res. II 49, 2,863-2,885.

Caldeira, K. Wickett, M.E, 2003. Anthropogenic carbon and ocean pH. Nature 425, 365

Caldeira, K., Wickett, M.E., 2005. Ocean model predictions of chemistry changes from carbon dioxide emissions to the atmosphere and ocean. J. Geophys. Res. 110, C09S04. http://dx.doi.org/10.1029/2004JC002671.

Canadell, J.G., Le Quere, C., Raupach, M.R., Field, C.B., Buitenhuis, E.T., Ciais, P., Conway, T.J., Gillett, N.P., Houghton, R.A., Marland, G., 2007. Contributions to accelerating atmospheric $\mathrm{CO}_{2}$ growth from economic activity, carbon intensity, and efficiency of natural sinks. Proc. Natl. Acad. Sci. 104, 18,866-18,870.

Clayton, T.D., Byrne, R.H., Breland, J.A., Feely, R.A., Millero, F.J., Campbell, D.M., Murphy, P.P., Lamb, M.F., 1995. The role of pH measurements in modern oceanic $\mathrm{CO}_{2}$-system characterizations: precision and thermodynamic consistency. Deep-Sea Res. I 42, 411-431.

Dickson, A.G., 1990. Standard potential of the reaction: $\mathrm{AgCl}(\mathrm{s})+1 / 2 \mathrm{H}_{2}(\mathrm{~g})=$ $\mathrm{Ag}(\mathrm{s})+\mathrm{HCl}(\mathrm{aq})$, and the standard acidity constant of the ion $\mathrm{HSO}_{4}^{-}$in synthetic seawater from 273.15 to $318.15 \mathrm{~K}$. J. Chem. Thermodyn. 22, 113-127.

Dickson, A.G., 1993. pH buffers for sea water media based on the total hydrogen ion concentration scale. Deep-Sea Res. A 40, 107-118.

Dickson, A.G., Riley, J., 1974. The effect of analytical error on the evaluation of the components of the aquatic carbon-dioxide system. Mar. Chem. 6, 77-85.

Dickson, A.G., Millero, F.J., 1987. A comparison of the equilibrium constants for the dissociation of carbonic acid in seawater media. Deep-Sea Res. I 34, 1,733-1,743.

Dickson, A.G., Sabine, C.L., Christian, J.R., 2007. Guide to best practices for ocean $\mathrm{CO}_{2}$ measurements. PICES Special Publication 3.

Dunne, J.P., Sarmiento, J.L., Gnanadesikan, A., 2007. A synthesis of global particle export from the surface ocean and cycling through the ocean interior and on the seafloor. Global Biogeochem. Cycles 21, GB4006. http://dx.doi.org/10.1029/2006GB002907.

Dyrssen, D.W., Uppstrom, L.R., 1974. The boron/chlorinity ratio in Baltic Sea water. Ambio 3 (1), 44-46

Fabry, V.J., Seibel, B.A., Feely, R.A., Orr, J.C., 2008. Impacts of ocean acidification on marine fauna and ecosystem processes. ICES J. Mar. Sci. 65, 414-432.

Fraga, F., Alvarez Salgado, X.A., 2005. On the variation of alkalinity during phytoplankton photosynthesis. Cienc. Mar 31 (4), 627-639.

Frankignoulle, M., Borges, A.V., 2001. European continental shelf as a significant sink for atmospheric carbon dioxide. Global Biogeochem. Cycles 15, 569-576.

Friis, K., Körtzinger, A., Wallace, D.W.R., 2003. The salinity normalization of marine inorganic carbon chemistry data. Geophys. Res. Lett. 30, 1085. http://dx.doi.org/10. 1029/2002GL015898.

Goyet, C., Poisson, A., 1989. New determination of carbonic acid dissociation constants in seawater as a function of temperature and salinity. Deep-Sea Res. I 36, 1,635-1,654.

Gran, G., 1952. Determination of the equivalence point in potentiometric titrations of seawater with hydrochloric acid. Oceanol. Acta 5, 209-218.

Grasshof, K., Ehrhardt, M., Kremling, K. (Eds.), 1983. Methods of seawater analysis. Verlag Chemie GmbH, Weinheim/Deerfield Beach, Florida, pp. 125-188.

Gripenberg, S., 1960. On the alkalinity of Baltic waters. ICES J. Mar. Sci. 26, 5-20.

Hansson, I., 1973. A new set of acidity constants for carbonic acid and boric acid in sea water. Deep-Sea Res. I 20, 461-478.

Hernandez-Ayon, J.M., Zirino, A., Dickson, A.G., Camiro-Vargas, T., Valenzuela-Espinoza, E., 2007. Estimating the contribution of organic bases from microalgae to the titration alkalinity in coastal seawaters. Limnol. Oceanogr. Methods 5, 225-232.

Hoppe, C.J.M., Langer, G., Rokitta, S.D., Wolf-Gladrow, D.A., Rost, B., 2012. Implications of observed inconsistencies in carbonate chemistry measurements for ocean acidification studies. Biogeosciences 9, 2401-2405.

Houghton, S.D., 1991. Coccolith sedimentation and transport in the North Sea. Mar. Geol. 99, 267-274

Johnson, K.M., Wills, K.D., Butler, D.B., Johnson, W.K., Wong, C.S., 1993. Coulometric total carbon dioxide analysis for marine studies: maximizing the performance of an automated gas extraction system and coulometric detector. Mar. Chem. 44, 167-187. 
Kempe, S., Pegler, K., 1991. Sinks and sources of $\mathrm{CO}_{2}$ in coastal seas: the North Sea. Tellus B 43, 224-235.

Khatiwala, S., Primeau, F., Hall, T., 2009. Reconstruction of the history of anthropogenic CO2 concentrations in the ocean. Nature 462, 346-349.

Kim, H.-C., Lee, K., 2009. Significant contribution of dissolved organic matter to seawater alkalinity. Geophys. Res. Lett. 36, L20603.

Koeve, W., Oschlies, A., 2012. Potential impact of DOC accumulation on $\mathrm{fCO}_{2}$ and carbonate ion computations in ocean acidification experiments. Biogeosciences 9, 3,787-3,798.

Körtzinger, A., Thomas, H., Schneider, B., Gronau, N., Mintrop, L., Duinker, J.C., 1996. At-sea intercomparison of two newly designed underway $\mathrm{pCO}_{2}$ systems - encouraging results. Mar. Chem. 52, 133-145.

Körtzinger, A., Mintrop, L., Wallace, D.W.R., Johnson, K.M., Neill, C., Tilbrook, B., Towler, P., Inoue, H.Y., Ishii, M., Shaffer, G., Torres Saavedra, R.F., Ohtaki, E., Yamashita, E., Poisson, A., Brunet, C., Schauer, B., Goyet, C., Eischeid, G., 2000. The international atsea intercomparison of $\mathrm{fCO}_{2}$ systems during the R/V Meteor Cruise 36/1 in the North Atlantic Ocean. Mar. Chem. 72, 171-192.

Kremling, K., 1967. Untersuchungen uber die chemische Zusammensetzung des Meerwassers der Ostsee II. Fruhjahr 1966. Kieler Meeresforsch. 26, 1-20.

Kremling, K., 1972. Untersuchungen ueber die chemische Zusammensetzung des Meerwassers aus der Ostsee. Kiel. Meeresforsch. XXVIII (H.2), 99-118.

Le Quéré, C., Moriarty, R., Andrew, R.M., Peters, G.P., Ciais, P., Friedlingstein, P. Jones, S.D., Sitch, S., Tans, P., Arneth, A., Boden, T.A., Bopp, L., Bozec, Y., Canadell, J.G., Chini, L.P., Chevallier, F., Cosca, C.E., Harris, I., Hoppema, M., Houghton, R.A., House, J.I., Jain, A.K., Johannessen, T., Kato, E., Keeling, R.F., Kitidis, V., Klein Goldeweijk, K., Koven, C., Landa, C.S., Landschützer, P., Lenton, A., Lima, I.D., Marland, G., Mathis, J.T., Metzl, N., Nojiri, Y., Olsen, A., Ono, T., Peng, S., Peters, W., Pfeil, B., Poulter, B., Raupach, M.R., Regnier, P., Rodenbeck, C., Saito, S., Salisbury, J.E., Schuster, U., Schwinger, J., Seferian, R., Segschneider, J., Steinhoff, T., Stocker, B.D., Sutton, A.J., Takahashi, T. Tilbrook, B., van der Werf, G.R., Viovy, N., Want, Y.-P., Wanninkhof, R., Wiltshire, A., Zeng, N., 2015. Global carbon budget 2014. Earth Syst. Sci. Data 7, 47-85.

Lee, K., Millero, F.J., Campbell, D.M., 1996. The reliability of the thermodynamic constants for the dissociation of carbonic acid in seawater. Mar. Chem. 55, 233-245.

Lee, K., Millero, F., Wanninkhof, R., 1997. The carbon dioxide system in the Atlantic Ocean. J. Geophys. Res. 102, 15,693-15,707.

Lee, K., Millero, F.J., Byrne, R.H., Feely, R.A., Wanninkhof, R., 2000. The recommended dissociation constants for carbonic acid in seawater. Geophys. Res. Lett. 27, 229-232.

Lee, K., Kim, T.W., Byrne, R.H., Millero, F.J., Feely, R.A., Liu, Y.M., 2010. The universal ratio of boron to chlorinity for the North Pacific and North Atlantic oceans. Geochim. Cosmochim. Acta 74, 1801-1811. http://dx.doi.org/10.1016/j.gca.2009.12.027.

Lewis, E.L., Wallace, D.W.R., 1998. Program developed for $\mathrm{CO}_{2}$ system calculations, ORNL/ CDIAC-105. Carbon dioxide information analysis center, Oak Ridge National Laboratory, U.S. Department of Energy, Oak Ridge.

Lueker, T.J., Dickson, A.G., Keeling, C.D., 2000. Ocean $\mathrm{pCO}_{2}$ calculated from dissolved inorganic carbon, alkalinity, and equations for $\mathrm{K}_{1}$ and $\mathrm{K}_{2}$ : validation based on laboratory measurements of $\mathrm{CO}_{2}$ in gas and seawater at equilibrium. Mar. Chem. 70, 105-119.

Mehrbach, C., Culberson, C.H., Hawley, J.E., Pytkowicz, R.M., 1973. Measurement of the apparent dissociation constants of carbonic acid in seawater at atmospheric pressure. Limnol. Oceanogr. 18, 897-907.

Millero, F.J., Byrne, R.H., Wanninkhof, R., Feely, R., Clayton, T., Murphy, P., Lamb, M.F., 1993. The internal consistency of $\mathrm{CO}_{2}$ measurements in the equatorial Pacific. Mar. Chem. 44, 269-280.

Millero, F.J., Pierrot, D., Lee, K., Wanninkhof, R., Feely, R., Sabine, C.L., Key, R.M., Takahashi, T., 2002. Dissociation constants for carbonic acid determined from field measurements. Deep-Sea Res. I 49, 1705-1723.

Millero, F.J., Graham, T.B., Huang, F., Bustos-Serrano, H., Pierrot, D., 2006. Dissociation constants of carbonic acid in seawater as a function of salinity and temperature. Mar. Chem. 100, 80-94.

Mojica-Prieto, F.J., Millero, F.J., 2002. The values of $\mathrm{pK}_{1}$ and $\mathrm{pK}_{2}$ for the dissociation of carbonic acid in seawater. Geochim. Cosmochim. Acta 66, 2,529-2,540.

Mosley, L.M., Peake, B.M., Hunter, K.A., 2010. Modelling of pH and inorganic carbon speciation in estuaries using the composition of the river and seawater end members. Environ. Model. Softw. 25, 1,658-1,663.

Muller, F.L.L., Bleie, B., 2008. Estimating the organic acid contribution to coastal seawater alkalinity by potentiometric titrations in a closed cell. Anal. Chim. Acta 619, 183-191.
Omar, A.M., Olsen, A., Johannessen, T., Hoppema, M., Thomas, H., Borges, A.V., 2010. Spatiotemporal variations of $\mathrm{fCO}_{2}$ in the North Sea. Ocean Sci. 6, 77-89. http://dx.doi.org/ 10.5194/os-6-77-2010

Osterroht, C., 1993. Extraction of dissolved fatty acids from sea water. Fresenius J. Anal. Chem. 345, 773-779.

Otto, L., Zimmerman, J.T.F., Furnes, G.K., Mork, M., Saetre, R., Becker, G., 1990. Review of the physical oceanography of the North Sea. Neth. J. Sea Res. 26, 161-238.

Park, K.P., 1969. Oceanic $\mathrm{CO}_{2}$ System: an evaluation of ten methods of investigation. Limnol. Oceanogr. 14, 179-186.

Provost, P., van Heuven, S., Soetaert, K., Laane, R.W.P.M., Middelburg, J.J., 2010. Seasonal and long-term changes in $\mathrm{pH}$ in the Dutch coastal zone. Biogeosciences 7, 3,869-3,878.

Prowe, A.E.F., Thomas, H., Pätsch, J., Kühn, W., Bozec, Y., Schiettecatte, L.-S., Borges, A.V., de Baar, H.J.W., 2009. Mechanisms controlling the air-sea $\mathrm{CO}_{2}$ flux in the North Sea. Cont. Shelf Res. 29, 1801-1808.

Raven, J., 2005. Ocean acidification due to increasing atmospheric carbon dioxide. Document No. 12/05. R. Soc. Lond. 60.

Ribas-Ribas, M., Rérolle, V.M.C., Bakker, D.C.E., Kitidis, V., Lee, G.A., Brown, I., Achterberg E.P., Tyrrell, T., 2014. Intercomparison of carbonate chemistry measurements on a cruise in northwestern European shelf seas. Biogeosciences 11, 4,339-4,355.

Roy, R.N., Roy, L.N., Vogel, K.M., Porter-Moore, C., Pearson, T., Good, C.E., Millero, F.J. Campbell, D.M., 1993. Determination of the ionization constants of carbonic acid in seawater. Mar. Chem. 44, 249-259.

Sabine, C.L., Feely, R.A., Gruber, N., Key, R.M., Lee, K., Bullister, J.L., Wanninkhof, R., Wong, C.S., Wallace, D.W.R., Tilbrook, B., Millero, F.J., Peng, T.-H., Kozyr, A., Ono, T., Rios, A.F., 2004. The oceanic sink for Anthropogenic $\mathrm{CO}_{2}$. Science 305, 367-371.

Salt, L.A., Thomas, H., Prowe, F.A.E., Borges, A.V., Bozec, Y., de Baar, H.J.W., 2013. Variability of North Sea pH and $\mathrm{CO} 2$ in response to North Atlantic Oscillation forcing. J. Geophys. Res. Biogeosci. 118, 1-9. http://dx.doi.org/10.1002/2013JG002306.

Schiettecatte, L.-S., Thomas, H., Bozec, Y., Borges, A.V., 2007. High temporal coverage of carbon dioxide measurements in the Southern Bight of the North Sea. Mar. Chem. 106, 161-173.

Stedmon, C.A., Osburn, C.L., Kragh, T., 2010. Tracing water mass mixing in the Baltic-North Sea transition zone using the optical properties of coloured dissolved organic matter. Estuar. Coast. Shelf Sci. 87, 156-162.

Thomas, H., Bozec, Y., Elkalay, K., de Baar, H.J.W., 2004. Enhanced open ocean storage of $\mathrm{CO}_{2}$ from shelf sea pumping. Science 304, 1005-1008.

Thomas, H., Bozec, Y., Elkalay, K., de Baar, H.J.W., Borges, A.V., Schiettecatte, L.-S., 2005 Controls of the surface water partial pressure of $\mathrm{CO}_{2}$ in the North Sea. Biogeosciences 2, 323-334.

Thomas, H., Schiettecatte, L.-S., Suykens, K., Koné, Y.J.M., Shadwick, E.H., Prowe, A.E.F., Bozec, Y., de Baar, H.J.W., Borges, A.V., 2009. Enhanced ocean carbon storage from anaerobic alkalinity generation in coastal sediments. Biogeosciences 6, 267-274.

Ulfsbo, A., Kulinski, K., Anderson, L.G., Turner, D.R., 2015. Modelling organic alkalinity in the Baltic Sea using a Humic-Pitzer approach. Mar. Chem. 168, 18-26.

Uppström, L.R., 1974. The boron/chlorinity ratio of deep-sea water from the Pacific Ocean. Deep-Sea Res. 21, 161-162.

van Heuven, S., Pierrot, D., Lewis, E., Wallace, D.W.R., 2011. MATLAB Program developed for $\mathrm{CO}_{2}$ system calculations. ORNL/CDIAC-105b. Carbon dioxide information analysis center. Oak Ridge National Laboratory, U.S. Department of Energy, Oak Ridge.

Wanninkhof, R., Lewis, E., Feely, R.A., Millero, F.J., 1999. The optimal carbonate dissociation constants for determining surface water $\mathrm{pCO}_{2}$ from alkalinity and total inorganic carbon. Mar. Chem. 65, 291-301.

Wolf-Gladrow, D.A., Zeebe, R.E., Klaas, C., Körtzinger, A., Dickson, A.G., 2007. Total alkalinity: the explicit conservative expression and its application to biogeochemical processes. Mar. Chem. 106, 287-300.

Wollast, R., 1998. Evaluation and comparison of the global carbon cycle in the coasta zone and in the open ocean. In: Brink, K.H., Robinson, A.R. (Eds.), The Sea. John Wiley \& Sons, New York, pp. 213-252.

Wootton, J.T., Pfister, C.A., Forester, J.D., 2008. Dynamic patterns and ecological impacts of declining ocean pH in a high-resolution multi-year dataset. Proc. Natl Acad. Sci. 105, $18,848-18,853$.

Zeebe, R.E., Wolf-Gladrow, D., 2001. $\mathrm{CO}_{2}$ in seawater: equilibrium, kinetics, isotopes. Elsevier, Amsterdam. 